TRANSACTIONS OF THE

AMERICAN MATHEMATICAL SOCIETY

Volume 359, Number 10, October 2007, Pages 5091-5115

S 0002-9947(07)04318-8

Article electronically published on April 24, 2007

\title{
MORSE DECOMPOSITIONS OF NONAUTONOMOUS DYNAMICAL SYSTEMS
}

\author{
MARTIN RASMUSSEN
}

\begin{abstract}
The global asymptotic behavior of dynamical systems on compact metric spaces can be described via Morse decompositions. Their components, the so-called Morse sets, are obtained as intersections of attractors and repellers of the system. In this paper, new notions of attractor and repeller for nonautonomous dynamical systems are introduced which are designed to establish nonautonomous generalizations of the Morse decomposition. The dynamical properties of these decompositions are discussed, and nonautonomous Lyapunov functions which are constant on the Morse sets are constructed explicitly. Moreover, Morse decompositions of one-dimensional and linear systems are studied.
\end{abstract}

\section{INTRODUCTION}

The study of isolated invariant sets of flows in the early 1970s has provided a great impetus to the development of the Conley Index Theory. Important classes of isolated invariant sets are given by attractors and repellers. Let $\Phi: \mathbb{R} \times X \rightarrow X$ be a flow on a compact metric space $X$. Then, a compact set $A \subset X$ is called an attractor of $\Phi$ if $A$ is invariant, i.e., $\Phi(t, A)=A$ for all $t \in \mathbb{R}$, and if $A$ is the $\omega$-limit set of some neighborhood $V$ of $A$, i.e., $A=\omega(V):=\bigcap_{t \geq 0} \overline{\Phi([t, \infty), V)}$. The set $V$ is called an isolating neighborhood of $A$. An invariant compact set $R \subset X$ is called a repeller if it is the $\alpha$-limit set of some neighborhood $W$ of $R$, i.e., $R=\alpha(W):=\bigcap_{t \leq 0} \overline{\Phi((-\infty, t], W)}$. The compactness of $X$ implies that for every repeller $R$, the corresponding set $R^{*}:=\{x \in X: \alpha(\{x\}) \cap R=\emptyset\}$ is an attractor. $\left(R^{*}, R\right)$ is called an attractor-repeller pair.

Given an attractor-repeller pair, we are able to understand the asymptotic behavior between the attractor and the repeller, since every point outside of this pair converges to the attractor in forward time and to the repeller in backward time. But what is going on inside the attractor and the repeller remains invisible. For a detailed analysis of the dynamics it is thus necessary to generalize the concept of an attractor-repeller pair. Therefore, one considers a finite sequence of repellers $X=R_{0} \supsetneq R_{1} \supsetneq R_{2} \supsetneq \cdots \supsetneq R_{n}=\emptyset$. Then, the so-called Morse sets $M_{i}:=R_{i}^{*} \cap R_{i-1}, i \in\{1, \ldots, n\}$, represent a more detailed view of the behavior

Received by the editors August 2, 2005 and, in revised form, December 1, 2005.

2000 Mathematics Subject Classification. Primary 34D05, 37B25, 37B55, 37C70; Secondary $34 \mathrm{D} 08$.

Key words and phrases. Attractor, attractor-repeller pair, Lyapunov function, Morse decomposition, Morse set, nonautonomous dynamical system, repeller.

This research was supported by the "Graduiertenkolleg: Nichtlineare Probleme in Analysis, Geometrie und Physik" (GK 283) financed by the DFG and the State of Bavaria.

(C)2007 American Mathematical Society 
of the dynamical system: Every point outside of these sets converges in forward as well as in backward time to one Morse set. The Morse sets together form a Morse decomposition $\left\{M_{1}, \ldots, M_{n}\right\}$ of $\Phi$.

For a discussion of elementary properties of Morse decompositions, we refer to the original work of Conley [8] (see also Colonius and KliEmann [6, Appendix B2], Rybakowski [18, Chapter 3], Robinson 17 and Akin [1). Recently, Ochs 16] used the notion of a weak attractor to construct Morse decompositions for random dynamical systems (see also Crauel, Duc and Siegmund 9]).

In this article, we want to go a step towards a nonautonomous generalization of this theory. For general nonautonomous dynamical systems acting on a compact metric space, we introduce suitable notions of attractor and repeller which allow the definition of an attractor-repeller pair and, moreover, lead to nonautonomous Morse decompositions. Since we are in a nonautonomous context, it makes a difference at which time we consider the system. Therefore, we introduce separate notions concerning the past and the future of the system.

This paper is organized as follows. In Section 2, we briefly recall some basic facts about nonautonomous dynamical systems. The nonautonomous generalizations of attractor and repeller are introduced in Section 3. Section 4 is devoted to the construction of attractor-repeller pairs. In Section 5] we define the nonautonomous counterparts of the Morse decomposition and discuss their basic dynamical properties. In Section 6] we show that there exist nonautonomous Lyapunov functions which are constant on the Morse sets and strictly decreasing along solutions outside of them. Finally, in Section 7 and Section 8 , we study Morse decompositions of one-dimensional systems and of linear flows on the projective space.

\section{Preliminaries}

As usual, we denote by $\mathbb{Z}$ and $\mathbb{R}$ the sets containing all integers and reals, respectively, and we define $\mathbb{Z}^{+}:=\mathbb{Z} \cap(0, \infty)$ and $\mathbb{R}^{+}:=(0, \infty)$. The set of all real $N \times N$ matrices is denoted by $\mathbb{R}^{N \times N}$. The $(N-1)$-sphere of the $\left(\mathbb{R}^{N},\|\cdot\|\right)$ is defined by $\mathbb{S}^{N-1}:=\left\{x \in \mathbb{R}^{N}:\|x\|=1\right\}$. Given a metric space $(X, d)$, we write $U_{\varepsilon}\left(x_{0}\right)=\left\{x \in X: d\left(x, x_{0}\right)<\varepsilon\right\}$ for the $\varepsilon$-neighborhood of a point $x_{0} \in X$ and $U_{\varepsilon}(A)=\bigcup_{x \in A} U_{\varepsilon}(x)$ for the $\varepsilon$-neighborhood of a set $A \subset X$. For arbitrary nonempty sets $A, B \subset X$ and $x \in X$, let $d(x, A):=\inf \{d(x, y): y \in A\}$ be the distance of $x$ to $A$ and $d(A \mid B):=\sup \{d(x, B): x \in A\}$ be the Hausdorff semidistance of $A$ and $B$. In addition, if $A$ and $B$ are empty, we define $d(A \mid B):=0$. The Hausdorff distance of $A$ and $B$ is defined by $d_{H}(A, B):=\max \{d(A \mid B), d(B \mid A)\}$.

The notion of a nonautonomous dynamical system emerged in the late $1990 \mathrm{~s}$ as an abstraction of both random dynamical systems (see, e.g., ARNOLD [2]) and continuous skew product flows (see, e.g., SELL [21, 22, 23]). The definition is given as follows.

Definition 2.1. A nonautonomous dynamical system (NDS for short) with a base set $P$, a locally compact metric space $(X, d)$ and a time $\mathbb{T}=\mathbb{R}$ or $\mathbb{T}=\mathbb{Z}$ consists of the following two ingredients:

(i) A model of the nonautonomy, given by a dynamical system $\theta: \mathbb{T} \times P \rightarrow P$, the so-called base flow, i.e., for all $t, s \in \mathbb{T}$ and $p \in P$, we have

$$
\theta(0, p)=p \quad \text { and } \quad \theta(t+s, p)=\theta(t, \theta(s, p)) .
$$


(ii) A model of the system under nonautonomous influence, given by a cocycle $\varphi: \mathbb{T} \times P \times X \rightarrow X$ over $\theta$, i.e., for all $t, s \in \mathbb{T}, p \in P$ and $\xi \in X$, we have

$$
\varphi(0, p, \xi)=\xi \quad \text { and } \quad \varphi(t+s, p, \xi)=\varphi(t, \theta(s, p), \varphi(s, p, \xi)),
$$

and the mapping $\varphi(\cdot, p, \cdot): \mathbb{T} \times X \rightarrow X$ is continuous for all $p \in P$.

For simplicity in notation, we write $\theta_{t} p$ instead of $\theta(t, p)$ and $\varphi(t, p) \xi$ instead of $\varphi(t, p, \xi)$.

Standard examples of nonautonomous dynamical systems are provided by nonautonomous differential equations $\dot{x}=f(t, x), \mathbb{T}=\mathbb{R}$, and nonautonomous difference equations $x_{n+1}=f\left(n, x_{n}\right), \mathbb{T}=\mathbb{Z}$, fulfilling conditions of global existence and uniqueness of solutions. The base set $P$ can simply be chosen to be $\mathbb{T}$ with the base flow $(t, s) \mapsto t+s ; \varphi(t, s, \xi)$ is the value at time $t+s$ of the solution fulfilling the initial condition $x(s)=\xi$. However, $P$ is then noncompact, which may cause difficulties. This can be avoided for a special class of right hand sides $f$ by considering the Bebutov flow on the hull of $f$ (see, e.g., Sell [23]). Apart from deterministic also random and stochastic differential and difference equations (see, e.g., ARNolD 2]) and some other types of equations generate nonautonomous dynamical systems.

A subset $M$ of the extended phase space $P \times X$ is called a nonautonomous set; we use the term $p$-fiber of $M$ for the set $M(p):=\{x \in X:(p, x) \in M\}, p \in P$. We call $M$ closed or compact if all $p$-fibers are closed or compact, respectively. Finally, a nonautonomous set $M$ is called invariant if $\varphi(t, p, M(p))=M\left(\theta_{t} p\right)$ for all $t \in \mathbb{T}$ and $p \in P$.

In addition to the NDS $(\theta, \varphi)$, we also consider the nonautonomous dynamical system under time-reversal, given by the base flow $(t, p) \mapsto \theta(-t, p)$ and the cocycle $(t, p, \xi) \mapsto \varphi(-t, p, \xi)$. We denote this NDS by $(\theta, \varphi)^{-1}$. Then, a nonautonomous set is invariant with respect to $(\theta, \varphi)$ if and only if it is invariant with respect to $(\theta, \varphi)^{-1}$.

\section{Nonautonomous attractors AND REPEllers}

In this section, we introduce new notions of attractor and repeller. Since we are in a nonautonomous context, we carry out the analysis with respect to the past and the future of the system.

Definition 3.1 (Past attractivity and repulsivity). Let $(\theta, \varphi)$ be an NDS, $A$ and $R$ be invariant and compact nonautonomous sets and $\mathcal{D}$ be a collection of nonautonomous sets.

(i) $A$ is called a $\mathcal{D}$-past attractor if for all $p \in P$ and $D \in \mathcal{D}$, we have

$$
\lim _{t \rightarrow \infty} d\left(\varphi\left(t, \theta_{-t} p\right) D\left(\theta_{-t} p\right) \mid A(p)\right)=0 .
$$

(ii) $A$ is called a past attractor if $A$ is a $\{D\}$-past attractor for some nonautonomous set $D$ fulfilling the following property: There exists an $\eta>0$ such that for all $p \in P$, there exists a $\tau>0$ with

$$
U_{\eta}\left(A\left(\theta_{-t} p\right)\right) \subset D\left(\theta_{-t} p\right) \quad \text { for all } t \geq \tau .
$$

(iii) $R$ is called a $\mathcal{D}$-past repeller if for all $p \in P$ and $D \in \mathcal{D}$, we have

$$
\lim _{t \rightarrow \infty} d\left(\varphi(-t, p) D(p) \mid R\left(\theta_{-t} p\right)\right)=0 .
$$


(iv) $R$ is called a past repeller if $R$ is a $\{D\}$-past repeller for some nonautonomous set $D$ fulfilling the following property: There exists an $\eta>0$ such that for all $p \in P$, there exists a $\tau>0$ with

$$
U_{\eta}\left(R\left(\theta_{-t} p\right)\right) \subset D\left(\theta_{-t} p\right) \text { for all } t \geq \tau .
$$

It follows directly from the definitions that the empty set is both a past attractor and a past repeller. If $X$ is compact, then $P \times X$ is also a past attractor and a past repeller.

\section{Remark 3.2 .}

(i) Definition 3.1 (ii) can be simplified as follows: $A$ is a past attractor if and only if there exists an $\eta>0$ such that $A$ is a $\{D\}$-past attractor with $D$ defined by $D(p):=U_{\eta}(A(p))$ for all $p \in P$.

(ii) The notion of a $\mathcal{D}$-past attractor coincides with the notion of a pullback attractor with respect to $\mathcal{D}$ used in the literature (see, e.g., KLOEDEN, KELLER and SCHMALFuss [14), with the difference that the so-called attraction universe $\mathcal{D}$ is supposed to contain the attractor. This additional condition guarantees uniqueness (with respect to $\mathcal{D}$ ) and, however, is fulfilled by a past attractor. Thus, a past attractor is a local form of a pullback attractor, i.e., it attracts a neighborhood of itself in the sense of a pullback attractor.

(iii) Every invariant and compact nonautonomous set of the differential equation $\dot{x}=x$ is a past repeller. Therefore, past repellers are not uniquely determined in general.

(iv) Past attractors and repellers are generalizations of autonomous attractors and repellers.

Before discussing the new definitions by means of a simple example, we introduce the notions of future attractors and repellers.

Definition 3.3 (Future attractivity and repulsivity). Let $(\theta, \varphi)$ be an NDS, $A$ and $R$ be invariant and compact nonautonomous sets and $\mathcal{D}$ be a collection of nonautonomous sets.

(i) $A$ is called a $\mathcal{D}$-future attractor if $A$ is a $\mathcal{D}$-past repeller of $(\theta, \varphi)^{-1}$.

(ii) $A$ is called a future attractor if $A$ is a past repeller of $(\theta, \varphi)^{-1}$.

(iii) $R$ is called a $\mathcal{D}$-future repeller if $R$ is a $\mathcal{D}$-past attractor of $(\theta, \varphi)^{-1}$.

(iv) $R$ is called a future repeller if $R$ is a past attractor of $(\theta, \varphi)^{-1}$.

Remark 3.4. In the following, we develop the theory of nonautonomous Morse decompositions only for the case of past attractivity and past repulsivity. Due to Definition 3.3. analogous results for the future of the system are easily obtained.

Example 3.5. The nonautonomous differential equation

$$
\dot{x}=a(t) x+b(t) x^{3}=: f(t, x)
$$

with continuous functions $a: \mathbb{R} \rightarrow \mathbb{R}$ and $b: \mathbb{R} \rightarrow(\gamma, \infty)$ for some $\gamma>0$ generates a nonautonomous dynamical system with $P=\mathbb{R}$ (see Section 21). For $t \in \mathbb{R}$ with $a(t) \geq 0, f(t, x)$ has the same sign as $x \in \mathbb{R}$. In case $a(t)<0$, we have 
$f\left(t, \pm \sqrt{-\frac{a(t)}{b(t)}}\right)=0$, and therefore, $f(t, x)$ has the opposite sign to $x$ in a vicinity of 0 . Thus, $\mathbb{R} \times\{0\}$ is a

(i) past attractor if $\liminf _{t \rightarrow-\infty}-\frac{a(t)}{b(t)}>0$,

(ii) past repeller if $\liminf _{t \rightarrow-\infty} a(t) \geq 0$,

(iii) future attractor if $\liminf _{t \rightarrow \infty}-\frac{a(t)}{b(t)}>0$,

(iv) future repeller if $\liminf _{t \rightarrow \infty} a(t) \geq 0$.

These conditions are only sufficient for stability of the trivial solution but not necessary. This example shows that, in general, the notions of past and future attractivity and repulsivity are independent of each other.

\section{Past attractor-Repeller Pairs}

Henceforth, we suppose that $(\theta, \varphi)$ is an NDS with base set $P$ and a compact metric space $(X, d)$. In this section, we want to analyze if for a given past repeller, there exists a corresponding past attractor and vice versa. Therefore, we first state an existence result for past attractors.

Theorem 4.1 (Existence of past attractors). Let $B$ be a compact nonautonomous set and $\mathcal{D}$ be a collection of nonautonomous sets with $B \in \mathcal{D}$. If for every $D \in \mathcal{D}$ and $p \in P$, there exists a $t^{*}>0$ such that

$$
\varphi\left(t, \theta_{-t} p\right) D\left(\theta_{-t} p\right) \subset B(p) \text { for all } t \geq t^{*},
$$

then there exists a $\mathcal{D}$-past attractor $A \subset B$, which is given by

$$
A(p):=\bigcap_{\tau \geq 0} \overline{\bigcup_{t \geq \tau} \varphi\left(t, \theta_{-t} p\right) B\left(\theta_{-t} p\right)} \quad \text { for all } p \in P .
$$

If, in addition, $A \subset D$ for some $D \in \mathcal{D}$, then $A$ is uniquely determined.

Proof. See Flandoli and Schmalfuss [10, Theorem 3.5].

Remark 4.2. In the literature (see, e.g., [10]), a nonautonomous set $B$ fulfilling (4.1) is called $\mathcal{D}$-absorbing.

Due to the Axiom of Choice, there exists a set $P^{*} \subset P$ such that $\left\{\theta_{t} p: t \in \mathbb{T}\right\} \cap P^{*}$ is a singleton for all $p \in P$. We write $P^{*}=P_{p}^{*} \cup P_{n}^{*}$ with $P_{p}^{*}$ containing all periodic points in $P^{*}$, i.e., $p^{*} \in P_{p}^{*}$ if and only if there exists a $\tau \in \mathbb{T}^{+}$with $p^{*}=\theta_{\tau} p^{*}$, and $P_{n}^{*}:=P^{*} \backslash P_{p}^{*}$.

Let $R$ be a past repeller. Thus, there exists an $\eta>0$ such that for every $p^{*} \in P^{*}$, there exists a $t^{*}\left(p^{*}\right)>0$ with

$$
\lim _{t \rightarrow \infty} d\left(\varphi\left(-t, \theta_{-\tau} p^{*}\right) U_{\eta}\left(R\left(\theta_{-\tau} p^{*}\right)\right) \mid R\left(\theta_{-\tau-t} p^{*}\right)\right)=0 \quad \text { for all } \tau \geq t^{*}\left(p^{*}\right) .
$$

For $\zeta \in(0, \eta]$, we define the compact nonautonomous set $B_{\zeta}$ by

$$
B_{\zeta}\left(\theta_{-t} p^{*}\right):=\left\{\begin{array}{cl}
X \backslash U_{\zeta}\left(R\left(\theta_{-t} p^{*}\right)\right) & : t \geq t^{*}\left(p^{*}\right) \\
X & : t<t^{*}\left(p^{*}\right)
\end{array} \quad \text { for all } p^{*} \in P_{n}^{*} \text { and } t \in \mathbb{T}\right.
$$

and

$$
B_{\zeta}\left(\theta_{-t} p^{*}\right):=X \backslash U_{\zeta}\left(R\left(\theta_{-t} p^{*}\right)\right) \text { for all } p^{*} \in P_{p}^{*} \text { and } t \in \mathbb{T} .
$$

Finally, we set $\mathcal{D}:=\left\{B_{\zeta}: \zeta \in(0, \eta]\right\}$. 
Theorem 4.3 (Existence of a past attractor-repeller pair). Let $R$ be a past repeller and $\mathcal{D}=\left\{B_{\zeta}: \zeta \in(0, \eta]\right\}$ be defined as above. Then, there exists a uniquely determined $\mathcal{D}$-past attractor $R^{*} \subset B_{\eta}$, which is also a past attractor. Furthermore, $R^{*}$ is the maximal past attractor outside $R$ in the following sense: Any past attractor $A \supsetneq R^{*}$ has nonempty intersection with $R$. We call $\left(R^{*}, R\right)$ a past attractor-repeller pair.

Proof. We show that the hypotheses of Theorem 4.1 (i) are fulfilled by setting $B:=B_{\eta}$. Thereto, let $\zeta \in(0, \eta]$ and $p \in P$. In case $B(p)=X$, the condition (4.1) certainly holds; otherwise, there exists a $p^{*} \in P^{*}$ and a $\tau \geq t^{*}\left(p^{*}\right)$ with $\theta_{-\tau} p^{*}=p$. Due to (4.2), there exists a $\hat{t} \geq 0$ with

$$
d\left(\varphi(-t, p) U_{\eta}(R(p)) \mid R\left(\theta_{-t} p\right)\right)<\frac{\zeta}{2} \quad \text { for all } t \geq \hat{t} .
$$

This means that $\varphi(-t, p) U_{\eta}(R(p)) \subset U_{\frac{\zeta}{2}}\left(R\left(\theta_{-t} p\right)\right)$ for all $t \geq \hat{t}$. Thus, we have

$$
\varphi(-t, p) B_{\eta}(p)=X \backslash \varphi(-t, p) U_{\eta}(R(p)) \supset B_{\zeta}\left(\theta_{-t} p\right) \text { for all } t \geq \hat{t} .
$$

This implies the desired relation $\varphi\left(t, \theta_{-t} p\right) B_{\zeta}\left(\theta_{-t} p\right) \subset B_{\eta}(p)$ for all $t \geq \hat{t}$. Therefore, Theorem 4.1 (i) guarantees the existence of a $\mathcal{D}$-past attractor $R^{*} \subset B_{\eta} . R^{*}$ is also a past attractor, since $B_{\frac{\eta}{2}} \in \mathcal{D}$ and $U_{\frac{\eta}{2}}\left(R^{*}(p)\right) \subset B_{\frac{\eta}{2}}(p)$ for all $p \in P$. Let $A \supsetneq R^{*}$ be another past attractor. Then, there exists a $p \in P$ with $A(p) \supsetneq R^{*}(p)$. We choose an $x \in A(p) \backslash R^{*}(p)$. Since $A \ni(p, x)$ is a past attractor, there exists an $\tilde{\eta}>0$ with

$$
\lim _{t \rightarrow \infty} d\left(\varphi\left(t, \theta_{-t} p\right) U_{\tilde{\eta}}(\varphi(-t, p) x) \mid A(p)\right)=0 .
$$

Due to $\lim _{t \rightarrow \infty} d\left(\varphi(-t, p) x, R\left(\theta_{-t} p\right)\right)=0$ (we will see this in Theorem 4.5 (ii)), there exists a sequence $\left\{y_{n}\right\}_{n \in \mathbb{N}}$ in $R(p)$ with

$$
\lim _{n \rightarrow \infty} d\left(y_{n}, A(p)\right)=0 .
$$

Since $R(p)$ and $A(p)$ are compact, this implies that their intersection is nonempty.

It is natural to ask if a past attractor implies the existence of a past repeller. The following example shows that this does not follow.

Example 4.4. The nonautonomous differential equation

$$
\dot{x}=f(t, x)
$$

with the function $f: \mathbb{R} \times[0,1] \rightarrow \mathbb{R}$, defined by

$$
f(t, x):=\left\{\begin{array}{cl}
|2 x-1|-1 & : t \geq 0 \text { and } x \in[0,1], \\
|2 x-1|-1 & : t<0 \text { and } x \in\left[0, \frac{1}{2}\right], \\
(2-2 t)\left(x-\frac{1}{2}\right)-1 & : t<0 \text { and } x \in\left[\frac{1}{2}, \frac{2-t}{2-2 t}\right], \\
0 & : t<0 \text { and } x \in\left(\frac{2-t}{2-2 t}, 1\right],
\end{array}\right.
$$

generates a nonautonomous dynamical system with $P=\mathbb{R}$ and $X=[0,1]$. The invariant nonautonomous set $A:=\mathbb{R} \times\{0\}$ is a past (as well as a future) attractor. Assume there exists a past repeller $A^{*} \subset \mathbb{R} \times(0,1]$. Due to the invariance of $A^{*}$, there exist $\gamma>\frac{1}{2}$ and $\tau_{1}<0$ with

$$
A^{*}(t) \subset(\gamma, 1] \text { for all } t \leq \tau_{1} .
$$


Thus, there exists a $\tau_{2}<\tau_{1}$ with

$$
A^{*}(s)=A^{*}(t) \text { and } f(t,[\gamma, 1])=\{0\} \quad \text { for all } t, s \leq \tau_{2} .
$$

This contradicts the fact that $A^{*}$ is a past repeller.

We now derive some properties of past attractor-repeller pairs.

Theorem 4.5 (Properties of past attractor-repeller pairs). Let $\left(R^{*}, R\right)$ be a past attractor-repeller pair. Then, the following statements hold:

(i) Isolation in the past. There exists a $\beta>0$ such that for all $p \in P$, there exists a $\tau>0$ with

$$
U_{\beta}\left(R^{*}\left(\theta_{-t} p\right)\right) \cap U_{\beta}\left(R\left(\theta_{-t} p\right)\right)=\emptyset \quad \text { for all } t \geq \tau .
$$

(ii) Backward convergence. Let $p \in P$ and $C \subset X \backslash R^{*}(p)$ be a compact set. Then, we have

$$
\lim _{t \rightarrow \infty} d\left(\varphi(-t, p) C \mid R\left(\theta_{-t} p\right)\right)=0 .
$$

(iii) Pullback convergence. For all $p \in P$ and all functions $\gamma: \mathbb{T}^{+} \rightarrow X$ with

$$
\liminf _{t \rightarrow \infty} d\left(\gamma(t), R\left(\theta_{-t} p\right)\right)>0
$$

we have

$$
\lim _{t \rightarrow \infty} d\left(\varphi\left(t, \theta_{-t} p\right) \gamma(t), R^{*}(p)\right)=0 .
$$

Proof. Let $\left(R^{*}, R\right)$ be a past attractor-repeller pair with $\eta$ and $\mathcal{D}$ defined as above.

(i) Theorem 4.3 implies $R^{*} \subset B_{\eta}$. The assertion follows by choosing $\beta:=\frac{\eta}{2}$.

(ii) Let $p \in P$ and $C \subset X \backslash R^{*}(p)$ be a compact set. Since $R^{*}$ is a $\mathcal{D}$-past attractor and thus a $\left\{B_{\eta}\right\}$-past attractor, there exists a $\tau>0$ such that

$$
C \cap \varphi\left(t, \theta_{-t} p\right) B_{\eta}\left(\theta_{-t} p\right)=\emptyset \text { for all } t \geq \tau .
$$

Hence, for all $t \geq \tau$, we have $\varphi(-t, p) C \cap B_{\eta}\left(\theta_{-t} p\right)=\emptyset$, and therefore, the relation $\lim _{t \rightarrow \infty} d\left(\varphi(-t, p) C, R\left(\theta_{-t} p\right)\right)=0$ holds.

(iii) We set $\zeta:=\frac{1}{2} \min \left\{\eta, \liminf _{t \rightarrow \infty} d\left(\gamma(t), R\left(\theta_{-t} p\right)\right)\right\}$ and see that there exists a $\tau>0$ with

$$
\gamma(t) \in B_{\zeta}\left(\theta_{-t} p\right) \text { for all } t \geq \tau .
$$

This finishes the proof, since $B_{\zeta} \in \mathcal{D}$ and $R^{*}$ is a $\mathcal{D}$-past attractor.

Theorem 4.3 implies that, given a past repeller $R$, the set $R^{*}$ is the uniquely determined past attractor outside of $R$ with the property of pullback convergence as described in Theorem 4.5 (iii). It is easy to see that such a uniqueness result is not valid for past repellers, i.e., it is possible that $\left(A, R_{1}\right)$ and $\left(A, R_{2}\right)$ are past attractor-repeller pairs with $R_{1} \neq R_{2}$. The following corollary says that in this case, $R_{1}$ and $R_{2}$ are converging to each other, when time tends to the past.

Proposition 4.6 (Form of nonuniqueness of past attractor-repeller pairs). Let $R_{1}$ and $R_{2}$ be past repellers with $R_{1}^{*}=R_{2}^{*}$. Then, we have

$$
\lim _{t \rightarrow \infty} d_{H}\left(R_{1}\left(\theta_{-t} p\right), R_{2}\left(\theta_{-t} p\right)\right)=0 \quad \text { for all } p \in P .
$$


Proof. Suppose there exists a $p \in P$ and sequences $\left\{t_{n}\right\}_{n \in \mathbb{N}}$ in $\mathbb{T}$ and $\left\{\gamma_{n}\right\}_{n \in \mathbb{N}}$ in $X$ with $\lim _{n \rightarrow \infty} t_{n}=\infty$ and $\gamma_{n} \in R_{1}\left(\theta_{-t_{n}} p\right)$ such that

$$
\liminf _{n \rightarrow \infty} d\left(\gamma_{n}, R_{2}\left(\theta_{-t_{n}} p\right)\right)>0 .
$$

Hence, Theorem 4.5 (iii), applied to the attractor-repeller pair $\left(R_{2}^{*}, R_{2}\right)$, implies that

$$
\lim _{n \rightarrow \infty} d\left(\varphi\left(t_{n}, \theta_{-t_{n}} p\right) \gamma_{n}, R_{2}^{*}(p)\right)=0 .
$$

Since $\varphi\left(t_{n}, \theta_{-t_{n}} p, \gamma_{n}\right) \in R_{1}(p)$ and $R_{1}$ and $R_{1}^{*}=R_{2}^{*}$ are compact nonautonomous sets, we have $R_{1}(p) \cap R_{1}^{*}(p) \neq \emptyset$. This is a contradiction.

\section{Past Morse Decompositions}

In this section, we generalize the notion of a past attractor-repeller pair by considering Morse decompositions.

Definition 5.1 (Past Morse decomposition). A family $\left\{M_{1}, M_{2}, \ldots, M_{n}\right\}$ of nonautonomous sets, the so-called Morse sets, is called a past Morse decomposition if the representation

$$
M_{i}=R_{i}^{*} \cap R_{i-1} \quad \text { for all } i \in\{1, \ldots, n\}
$$

holds with past repellers

$$
P \times X=R_{0} \supsetneq R_{1} \supsetneq \cdots \supsetneq R_{n}=\emptyset
$$

fulfilling $R_{0}^{*} \subsetneq R_{1}^{*} \subsetneq \cdots \subsetneq R_{n}^{*}$.

Remark 5.2. Let $(A, R)$ be a past attractor-repeller pair such that $\emptyset \subsetneq A \subsetneq P \times X$. Then, $\{A, R\}$ is a past Morse decomposition.

Proposition 5.3 (Basic properties of past Morse decompositions). The Morse sets of a past Morse decomposition $\left\{M_{1}, \ldots, M_{n}\right\}$ are nonempty, invariant, pairwise disjoint and isolated in the past, i.e., there exists a $\beta>0$ such that for all $1 \leq i<$ $j \leq n$ and all $p \in P$, there exists a $\tau>0$ with

$$
U_{\beta}\left(M_{i}\left(\theta_{-t} p\right)\right) \cap U_{\beta}\left(M_{j}\left(\theta_{-t} p\right)\right)=\emptyset \quad \text { for all } t \geq \tau .
$$

Proof. Let $M_{i}=R_{i}^{*} \cap R_{i-1}$ be a Morse set. Since $R_{i-1}^{*} \subsetneq R_{i}^{*}$, we can choose a $p \in P$ and an $x \in R_{i}^{*}(p) \backslash R_{i-1}^{*}(p)$. Since $R_{i}^{*} \ni(p, x)$ is a past attractor, there exists an $\eta>0$ with

$$
\lim _{t \rightarrow \infty} d\left(\varphi\left(t, \theta_{-t} p\right) U_{\eta}(\varphi(-t, p) x) \mid R_{i}^{*}(p)\right)=0 .
$$

Due to $\lim _{t \rightarrow \infty} d\left(\varphi(-t, p) x, R_{i-1}\left(\theta_{-t} p\right)\right)=0$ (cf. Theorem 4.5 (ii)), there exists a sequence $\left\{y_{n}\right\}_{n \in \mathbb{N}}$ in $R_{i-1}(p)$ with

$$
\lim _{n \rightarrow \infty} d\left(y_{n}, R_{i}^{*}(p)\right)=0 .
$$

Since $R_{i-1}(p)$ and $R_{i}^{*}(p)$ are compact, this implies that $M_{i}=R_{i}^{*} \cap R_{i-1} \neq \emptyset$. Furthermore, $M_{i}$ is the intersection of two invariant nonautonomous sets and thus invariant. Choose another Morse set $M_{j}=R_{j}^{*} \cap R_{j-1}$. W.l.o.g., we assume $j>i$. Then, we get

$$
M_{i} \cap M_{j}=R_{i}^{*} \cap R_{i-1} \cap R_{j}^{*} \cap R_{j-1}=R_{i-1}^{*} \cap R_{j-1} \subset R_{j-1}^{*} \cap R_{j-1}=\emptyset .
$$

The fact that the Morse sets are isolated in the past is an easy consequence of Theorem 4.5 (i). 
As in the autonomous case, past Morse decompositions are not uniquely determined.

Definition 5.4. We say that the past Morse decomposition $\left\{M_{1}, \ldots, M_{n}\right\}$ is finer than the past Morse decomposition $\left\{\tilde{M}_{1}, \ldots, \tilde{M}_{m}\right\}$ if

$$
\lim _{t \rightarrow \infty} d\left(\bigcup_{i=1}^{n} M_{i}\left(\theta_{-t} p\right) \mid \bigcup_{i=1}^{m} \tilde{M}_{i}\left(\theta_{-t} p\right)\right)=0 \quad \text { for all } p \in P .
$$

Remark 5.5.

(i) This definition is a generalization of the notion of a finer (autonomous) Morse decomposition. In the autonomous case, a Morse decomposition $\left\{M_{1}, \ldots, M_{n}\right\}$ is called finer than the Morse decomposition $\left\{\tilde{M}_{1}, \ldots, \tilde{M}_{m}\right\}$ if for all $j \in\{1, \ldots, m\}$, there exists an $i \in\{1, \ldots, n\}$ such that $M_{i} \subset \tilde{M}_{j}$ (see, e.g., Colonius and Kliemann [6, p. 542]). It is easy to see that this is equivalent to

$$
d\left(\bigcup_{i=1}^{n} M_{i} \mid \bigcup_{i=1}^{m} \tilde{M}_{i}\right)=0 .
$$

The additional limit in our nonautonomous context is motivated by Proposition 4.6 .

(ii) There are different forms of nonuniqueness for the Morse sets. As seen in Proposition 4.6. two past attractor-repeller pairs are converging to each other in case the past attractors are equal. One can find examples to show that such a (weak) form of nonuniqueness is not valid for arbitrary Morse decompositions (i.e., those consisting of more than two sets). However, in the special cases of one-dimensional and linear systems (cf. Section 7 and Section 8), one obtains similar results as in Proposition 4.6] (see Proposition 7.2 and Proposition 8.6).

The following theorem shows that the Morse sets are important for the asymptotic behavior of the nonautonomous dynamical system.

Theorem 5.6 (Dynamical properties of past Morse decompositions). Pullback convergence. Let $\left\{M_{1}, \ldots, M_{n}\right\}$ be a past Morse decomposition obtained by the finite sequence of past repellers $R_{0} \supset \cdots \supset R_{n}$. Then, for all $p \in P$ and all functions $\gamma: \mathbb{T}^{+} \rightarrow X$ with

$$
\liminf _{t \rightarrow \infty} d\left(\gamma(t), \bigcup_{j=1, \ldots, n} \partial R_{j}\left(\theta_{-t} p\right)\right)>0,
$$

we have

$$
\lim _{t \rightarrow \infty} d\left(\varphi\left(t, \theta_{-t} p\right) \gamma(t), \bigcup_{j=1, \ldots, n} M_{j}(p)\right)=0 .
$$

Proof. We assume w.l.o.g. that there exists an $i \in\{1, \ldots, n\}$ with

$$
\gamma(t) \in R_{i-1}\left(\theta_{-t} p\right) \text { and } \gamma(t) \notin R_{i}\left(\theta_{-t} p\right) \text { for all } t>0 .
$$

Then, $\liminf _{t \rightarrow \infty} d\left(\gamma(t), \partial R_{i}\left(\theta_{-t} p\right)\right)>0$ yields $\liminf _{t \rightarrow \infty} d\left(\gamma(t), R_{i}\left(\theta_{-t} p\right)\right)>0$. Therefore, Theorem 4.5 implies that

$$
\lim _{t \rightarrow \infty} d\left(\varphi\left(t, \theta_{-t} p\right) \gamma(t), R_{i}^{*}(p)\right)=0 .
$$


Assume that there exist an $\varepsilon>0$ and a sequence $\left\{t_{n}\right\}_{n \in \mathbb{N}}$ in $\mathbb{T}^{+}$with $\lim _{n \rightarrow \infty} t_{n}=$ $\infty$ and

$$
d\left(\varphi\left(t_{n}, \theta_{-t_{n}} p\right) \gamma\left(t_{n}\right), M_{i}(p)\right) \geq \varepsilon \quad \text { for all } n \in \mathbb{N} .
$$

W.l.o.g., the sequence $\left\{\varphi\left(t_{n}, \theta_{-t_{n}} p\right) \gamma\left(t_{n}\right)\right\}_{n \in \mathbb{N}}$ in $R_{i-1}(p)$ is convergent with limit $x_{0} \in R_{i-1}(p)\left(R_{i-1}(p)\right.$ is compact). Moreover, $x_{0} \in R_{i}^{*}(p)$, since (5.1) holds and $R_{i}^{*}(p)$ is compact. Thus, $x_{0} \in M_{i}(p)=R_{i}^{*}(p) \cap R_{i-1}(p)$. This contradicts (5.2) and finishes the proof of this theorem.

Remark 5.7. In contrast to past attractor-repeller pairs, the backward convergence condition as described in Theorem 4.5 does not hold for arbitrary past Morse decompositions. However, in the special cases of one-dimensional and linear systems (cf. Section 7 and Section 8), one obtains similar results as in Theorem 4.5 (see Theorem 7.1 and Theorem 8.5).

In case the backward convergence holds, one is able to prove the following uniqueness result concerning the past attractors.

Proposition 5.8. Let $\left\{M_{1}, \ldots, M_{n}\right\}$ be a past Morse decomposition obtained by the finite sequence of past repellers $R_{0} \supset \cdots \supset R_{n}$. We assume that the backward convergence holds, i.e., for all $(p, x) \in P \times X$, there exists an $i \in\{1, \ldots, n\}$ with

$$
\lim _{t \rightarrow \infty} d\left(\varphi(-t, p) x, M_{i}\left(\theta_{-t} p\right)\right)=0 .
$$

Then, the representation

$$
R_{i}^{*}=\left\{(p, x): \lim _{t \rightarrow \infty} d\left(\varphi(-t, p) x, \bigcup_{j=1, \ldots, i} M_{j}\left(\theta_{-t} p\right)\right)=0\right\} \quad \text { for all } i \in\{1, \ldots, n\}
$$

holds, i.e., the past attractors of a past Morse decomposition are uniquely determined.

Proof. ( $\subseteq)$ Let $(p, x) \in R_{i}^{*}$. We choose $j \in\{1, \ldots, n\}$ such that

$$
0=\lim _{t \rightarrow \infty} d\left(\varphi(-t, p) x, M_{j}\left(\theta_{-t} p\right)\right)=\lim _{t \rightarrow \infty} d\left(\varphi(-t, p) x, R_{j-1}\left(\theta_{-t} p\right)\right) .
$$

The assumption $j>i$ leads to $\lim _{t \rightarrow \infty} d\left(\varphi(-t, p) x, R_{i}\left(\theta_{-t} p\right)\right)=0$. This contradicts Theorem 4.5 (i), since $\varphi(-t, p) x \in R_{i}^{*}\left(\theta_{-t} p\right)$ for all $t \in \mathbb{T}$.

() Let $(p, x) \in(P \times X) \backslash R_{i}^{*}$. Then, Theorem 4.5 (ii) implies

$$
\lim _{t \rightarrow \infty} d\left(\varphi(-t, p) x, R_{i}\left(\theta_{-t} p\right)\right)=0 .
$$

The assumption $\lim _{t \rightarrow \infty} d\left(\varphi(-t, p) x, \bigcup_{j=1, \ldots, i} M_{j}\left(\theta_{-t} p\right)\right)=0$ leads to

$$
\lim _{t \rightarrow \infty} d\left(\varphi(-t, p) x, R_{i}^{*}\left(\theta_{-t} p\right)\right)=0,
$$

since $M_{j} \subset R_{i}^{*}$ for $j \in\{1, \ldots, i\}$. Due to Theorem 4.5)(i), this contradicts (5.3).

\section{LyapunOV FUnCTIONS FOR PAST MORSE DECOMPOSITIONS}

In this section, we obtain explicitly nonautonomous Lyapunov functions which are constant on the Morse sets and which strictly decrease outside them. We use a similar construction as in CONLEY [8, $\S 5$ and $\S 6$ of Chapter II] (see also Franks [11, $\S 1$ ], Robinson [17, Chapter X] and NorTon [15]), which has also been adapted in Kloeden [12, 13] and Arnold and Schmalfuss [3] in the nonautonomous setting. 
We first treat the case that the past Morse decomposition is given by a past attractor-repeller pair.

Lemma 6.1. Let $\left(R^{*}, R\right)$ be a past attractor-repeller pair. Then, there exists a function $L: P \times X \rightarrow[0,1]$ which is continuous with respect to $x \in X$ such that $\left.L\right|_{R^{*}} \equiv 0,\left.L\right|_{R} \equiv 1$ and

$$
L\left(\theta_{t} p, \varphi(t, p) x\right)<L(p, x) \quad \text { for all } t>0 \text { and }(p, x) \in(P \times X) \backslash\left(R^{*} \cup R\right)
$$

is satisfied.

Proof. We define the function $V: P \times X \rightarrow[0,1]$ by

$$
V(p, x):=\frac{d\left(x, R^{*}(p)\right)}{d\left(x, R^{*}(p)\right)+d(x, R(p))} \text { for all }(p, x) \in P \times X .
$$

This function is continuous with respect to $x \in X$ and fulfills $\left.V\right|_{R^{*}} \equiv 0,\left.V\right|_{R} \equiv 1$, but is not necessarily decreasing along solutions. Therefore, we define by

$$
V^{*}(p, x):=\inf _{s \geq 0} V\left(\theta_{-s} p, \varphi(-s, p) x\right) \quad \text { for all }(p, x) \in P \times X
$$

a function $V^{*}: P \times X \rightarrow[0,1]$ which obviously satisfies $\left.V^{*}\right|_{R^{*}} \equiv 0,\left.V^{*}\right|_{R} \equiv 1$ and $V^{*}\left(\theta_{t} p, \varphi(t, p) x\right) \leq V^{*}(p, x)$ for all $t \geq 0$ and $(p, x) \in P \times X$. To prove that $V^{*}(p, \cdot)$ is continuous for all $p \in P$, we first choose $\xi \in X \backslash R^{*}(p)$ and $\varepsilon>0$. Then, there exists a $\hat{\delta}>0$ such that $C:=\overline{U_{\hat{\delta}}(\xi)} \subset X \backslash R^{*}(p)$. It follows that

$$
\lim _{s \rightarrow \infty} \inf _{x \in C} V\left(\theta_{-s} p, \varphi(-s, p) x\right)=1,
$$

since $\lim _{s \rightarrow \infty} d\left(\varphi(-s, p) C, R\left(\theta_{-s} p\right)\right)=0($ cf. Theorem 4.5 (ii)) and there exist a $\beta>$ 0 and an $\hat{s}>0$ such that $d\left(\varphi(-s, p) C, R^{*}\left(\theta_{-s} p\right)\right) \geq \frac{\beta}{2}$ for all $s \geq \hat{s}$ (cf. Theorem 4.5 (i), (ii)). Thus, there exists an $s_{0}>0$ such that

$$
\inf _{x \in C} V\left(\theta_{-s} p, \varphi(-s, p) x\right)>1-\varepsilon \text { for all } s \geq s_{0} .
$$

Due to the continuity of $V(\theta(\cdot, p), \varphi(\cdot, p, \cdot)): \mathbb{T} \times X \rightarrow \mathbb{R}$, there exists a $\delta \in(0, \hat{\delta})$ such that

$\left|V\left(\theta_{-s} p, \varphi(-s, p) \xi\right)-V\left(\theta_{-s} p, \varphi(-s, p) x\right)\right|<\varepsilon \quad$ for all $x \in U_{\delta}(\xi)$ and $0 \leq s \leq s_{0}$.

This implies that $V^{*}(p, \cdot)$ is continuous in $\xi \notin R^{*}(p)$. The continuity of $V^{*}(p, \cdot)$ in $\xi \in R^{*}(p)$ follows directly from the continuity of $V$. We note that $V$ is not strictly decreasing along solutions in $(P \times X) \backslash\left(R^{*} \cup R\right)$. Therefore, we define $L$ to be a weighted average of $V^{*}$ over the backward solution:

$$
L(p, x):=\int_{0}^{\infty} e^{-s} V^{*}\left(\theta_{-s} p, \varphi(-s, p) x\right) d s \quad \text { for all }(p, x) \in P \times X .
$$

This function is obviously continuous with respect to $x \in X$, and we have

$$
\begin{aligned}
L\left(\theta_{t} p, \varphi(t, p, x)\right) & =\int_{0}^{\infty} e^{-s} V^{*}\left(\theta_{-s} \theta_{t} p, \varphi\left(-s, \theta_{t} p\right) \varphi(t, p) x\right) d s \\
& =\int_{0}^{\infty} e^{-s} V^{*}\left(\theta_{t} \theta_{-s} p, \varphi\left(t, \theta_{-s} p\right) \varphi(-s, p) x\right) d s \\
& \leq \int_{0}^{\infty} e^{-s} V^{*}\left(\theta_{-s} p, \varphi(-s, p) x\right) d s=L(p, x) .
\end{aligned}
$$

To prove that this function is also strictly decreasing along solutions in $(P \times X) \backslash$ $\left(R^{*} \cup R\right)$, we assume that $L\left(\theta_{t} p, \varphi(t, p) x\right)=L(p, x)$ for some $t>0$ and $x \in(P \times X) \backslash$ 
$\left(R^{*} \cup R\right)$. Then, $V^{*}\left(\theta_{-s} p, \varphi(-s, p) x\right)=V^{*}\left(\theta_{t-s} p, \varphi(t-s, p) x\right)$ for all $s \geq 0$. This is impossible, since $V^{*}(p, x) \in(0,1)$ and $\lim _{s \rightarrow \infty} V^{*}\left(\theta_{-s} p, \varphi(-s, p, x)\right)=0$.

In the next theorem, we extend the Lyapunov function from past attractorrepeller pairs to past Morse decompositions.

Theorem 6.2 (Lyapunov functions for past Morse decompositions). We consider a past Morse decomposition $\left\{M_{1}, \ldots, M_{n}\right\}$. Then, there exists a function $L: P \times$ $X \rightarrow[0,1]$ which is continuous with respect to $x \in X$ such that $\left.L\right|_{M_{i}} \equiv \frac{i-1}{n-1}$ for $i \in\{1, \ldots, n\}$ and

$$
L\left(\theta_{t} p, \varphi(t, p) x\right)<L(p, x) \quad \text { for all } t>0 \text { and }(p, x) \in(P \times X) \backslash\left(M_{1} \cup \cdots \cup M_{n}\right)
$$

is satisfied.

Proof. Let $P \times X=R_{0} \supsetneq R_{1} \supsetneq \cdots \supsetneq R_{n}=\emptyset$ be the sequence of past repellers leading to the given past Morse decomposition, i.e.,

$$
M_{i}=R_{i}^{*} \cap R_{i-1} \quad \text { for all } i \in\{1, \ldots, n\} .
$$

Furthermore, let $L_{i}, i \in\{1, \ldots, n-1\}$, be the Lyapunov function corresponding to the past attractor-repeller pair $\left(R_{i}^{*}, R_{i}\right)$ as introduced in Lemma 6.1] We define

$$
L(p, x):=\frac{1}{n-1} \sum_{i=1}^{n-1} L_{i}(p, x) \text { for all }(p, x) \in P \times X .
$$

Choose $(p, x) \in M_{i}$ arbitrarily, and let $j \in\{1, \ldots, n-1\}$. Then, $(p, x) \in R_{j}$ if and only if $j \in\{1, \ldots, i-1\}$, and $(p, x) \in R_{j}^{*}$ if and only if $j \in\{i, \ldots, n-1\}$. This implies $\left.L\right|_{M_{i}} \equiv \frac{i-1}{n-1}$ for all $i \in\{1, \ldots, n\}$. Now choose $(p, x) \in(P \times X) \backslash\left(M_{1} \cup \cdots \cup\right.$ $\left.M_{n}\right)$. Then, there exists a $j \in\{1, \ldots, n\}$ with $(p, x) \notin R_{j}^{*} \cup R_{j}$. This means that $L_{j}\left(\theta_{t} p, \varphi(t, p) x\right)<L_{j}(p, x)$ for all $t>0$ and finishes the proof of this theorem.

\section{Past Morse decompositions in dimension one}

In this section, we study past Morse decompositions of nonautonomous dynamical systems whose phase space is a compact interval. As we will see, in this special case, we get stronger results concerning the convergence behavior of the system and the nonuniqueness of the Morse sets.

Let $\mathbb{I} \subset \mathbb{R}$ be a compact interval and $(\theta: \mathbb{T} \times P \rightarrow P, \varphi: \mathbb{T} \times P \times \mathbb{I} \rightarrow \mathbb{I})$ be a nonautonomous dynamical system.

Theorem 7.1 (Dynamical properties of past Morse decompositions in dimension one). Let $\left\{M_{1}, \ldots, M_{n}\right\}$ be a past Morse decomposition obtained by the finite sequence of past repellers $R_{0} \supset \cdots \supset R_{n}$. Then, the following statements hold:

(i) Pullback convergence. For all $p \in P$ and all functions $\gamma: \mathbb{T}^{+} \rightarrow \mathbb{I}$ with

$$
\liminf _{t \rightarrow \infty} d\left(\gamma(t), \bigcup_{j=1, \ldots, n} \partial R_{j}\left(\theta_{-t} p\right)\right)>0,
$$

we have

$$
\lim _{t \rightarrow \infty} d\left(\varphi\left(t, \theta_{-t} p\right) \gamma(t), \bigcup_{j=1, \ldots, n} M_{j}(p)\right)=0 .
$$


(ii) Backward convergence. For all $(p, x) \in P \times \mathbb{I}$, there exists an $i \in\{1, \ldots, n\}$ with

$$
\lim _{t \rightarrow \infty} d\left(\varphi(-t, p) x, M_{i}\left(\theta_{-t} p\right)\right)=0 .
$$

Proof. (i) This assertion is also valid for general past Morse decompositions and was proved in Theorem 5.6 .

(ii) Choose $(p, x) \in P \times \mathbb{I}$ arbitrarily. Then, there exists an $i \in\{1, \ldots, n\}$ such that

$$
x \in R_{i}^{*}(p) \quad \text { and } \quad x \notin R_{i-1}^{*}(p) .
$$

In case $x \in R_{i-1}(p)$, the asserted limit relation follows, since then $x \in M_{i}(p)$ and $M_{i}$ is invariant. We therefore assume $x \notin R_{i-1}(p)$ from now on. Due to the topology of $\mathbb{I}, \varphi$ is order preserving in the following sense: For fixed $t \in \mathbb{T}$, exactly one of the following two statements holds:

- $y_{1}<y_{2}$ implies $\varphi(t, p) y_{1}<\varphi(t, p) y_{2}$,

- $y_{1}<y_{2}$ implies $\varphi(t, p) y_{1}>\varphi(t, p) y_{2}$.

Since $\lim _{t \rightarrow \infty} d\left(\varphi(-t, p) x, R_{i-1}\left(\theta_{-t} p\right)\right)=0$ (cf. Theorem 4.5 (ii)), this implies that there exists a $y \in R_{i-1}(p)$ such that

$$
\lim _{t \rightarrow \infty}|\varphi(-t, p) x-\varphi(-t, p) y|=0 .
$$

Because $R_{i}^{*}$ is a past attractor, there exists an $\eta>0$ such that

$$
R_{i}^{*}(p)=\bigcap_{\tau \geq 0} \bigcup_{t \geq \tau} \varphi\left(t, \theta_{-t} p\right) U_{\eta}\left(R_{i}^{*}\left(\theta_{-t} p\right)\right)=: \limsup _{t \rightarrow \infty} \varphi\left(t, \theta_{-t} p\right) U_{\eta}\left(R_{i}^{*}\left(\theta_{-t} p\right)\right)
$$

(cf. Theorem 4.1 the closure is not needed here). This implies that

$$
\limsup _{t \rightarrow \infty} \varphi\left(t, \theta_{-t} p\right) U_{\eta}(\varphi(-t, p) x) \subset R_{i}^{*}(p) .
$$

Due to (7.1), this leads to $y \in R_{i}^{*}(p)$. Hence, $y \in M_{i}^{*}(p)$, and this finishes the proof of this theorem.

In our special situation, we can generalize Proposition 4.6,

Proposition 7.2 (Form of nonuniqueness of the Morse sets). Let $\left\{M_{1}, \ldots, M_{n}\right\}$ and $\left\{\hat{M}_{1}, \ldots, \hat{M}_{n}\right\}$ be past Morse decompositions obtained by the finite sequences of past repellers $R_{0} \supset \cdots \supset R_{n}$ and $\hat{R}_{0} \supset \cdots \supset \hat{R}_{n}$. We assume that

$$
R_{i}^{*}=\hat{R}_{i}^{*} \quad \text { for all } i \in\{1, \ldots, n-1\} \text {. }
$$

Then, the relation

$$
\lim _{t \rightarrow \infty} d_{H}\left(M_{i}\left(\theta_{-t} p\right), \hat{M}_{i}\left(\theta_{-t} p\right)\right)=0 \quad \text { for all } i \in\{1, \ldots, n\} \text { and } p \in P
$$

is fulfilled.

Proof. Choose $i \in\{1, \ldots, n\}$ and $p \in P$ arbitrarily. W.l.o.g., we only show the relation

$$
\lim _{t \rightarrow \infty} d\left(M_{i}\left(\theta_{-t} p\right), \hat{M}_{i}\left(\theta_{-t} p\right)\right)=0 .
$$

We define $[p]:=\left\{\theta_{t} p: t \in \mathbb{T}\right\}$. The proof is divided into three steps.

Step 1. There exists a past repeller $\bar{R}_{i-1} \supset R_{i-1}$ with $\bar{R}_{i-1}^{*}=R_{i-1}^{*}$ such that

$$
\bar{M}_{i}(\bar{p}):=R_{i}^{*}(\bar{p}) \cap \bar{R}_{i-1}(\bar{p}) \quad \text { for all } \bar{p} \in[p]
$$

has only finitely many connected components. 
Since $R_{i}^{*}$ is a past attractor, there exists an $\eta>0$ such that

$$
R_{i}^{*}(p)=\limsup _{t \rightarrow \infty} \varphi\left(t, \theta_{-t} p\right) U_{\eta}\left(R_{i}^{*}\left(\theta_{-t} p\right)\right)
$$

(cf. proof of Theorem 7.1). Since $\varphi$ is continuous and $U_{\eta}\left(R_{i}^{*}\left(\theta_{-t} p\right)\right)$ has only finitely many connected components, this implies that $R_{i}^{*}$ has only finitely many connected components. Because $R_{i-1}$ is a past repeller, there exists a $\beta>0$ such that

$$
\lim _{t \rightarrow \infty} d\left(\varphi(-t, p) U_{\beta}\left(R_{i-1}(p)\right) \mid R_{i-1}\left(\theta_{-t} p\right)\right)=0 .
$$

Hence, the nonautonomous set $\bar{R}_{i-1}$, defined by

$$
\bar{R}_{i-1}(\bar{p}):=\left\{\begin{array}{cll}
\varphi(t, p) \overline{U_{\frac{\beta}{2}}\left(R_{i-1}(p)\right)} & : \bar{p}=\theta_{t} p \quad \text { for some } t \in \mathbb{T}, \\
R_{i-1}(\bar{p}) & : \bar{p} \notin[p],
\end{array}\right.
$$

is also a past repeller fulfilling $\bar{R}_{i-1}^{*}=R_{i-1}^{*}$. Moreover, for $\bar{p} \in[p]$, the set $\bar{R}_{i-1}(\bar{p})$ has only finitely many connected components, since $\varphi$ is continuous and $\overline{U_{\frac{\beta}{2}}\left(R_{i-1}(p)\right)}$ has only finitely many connected components. This implies the assertion.

Step 2. For all connected components $C$ of $\bar{M}_{i}(p)$, we have

$$
\lim _{t \rightarrow \infty} d\left(\varphi(-t, p) C \mid \hat{M}_{i}\left(\theta_{-t} p\right)\right)=0 .
$$

Let $C=\left[c_{1}, c_{2}\right]$ be a connected component of $\bar{M}_{i}(p)$, and choose $\varepsilon>0$ arbitrarily. Due to Theorem 7.1 (ii), there exists a $\tau_{1} \geq 0$ such that we have

$$
d\left(\varphi(-t, p) c_{j}, \hat{M}_{i}\left(\theta_{-t} p\right)\right) \leq \frac{\varepsilon}{2} \quad \text { for all } t \geq \tau_{1} \text { and } j \in\{1,2\} .
$$

Furthermore, because of Proposition 4.6 , there exists a $\tau_{2} \geq \tau_{1}$ with

$$
d_{H}\left(\bar{R}_{i-1}\left(\theta_{-t} p\right), \hat{R}_{i-1}\left(\theta_{-t} p\right)\right) \leq \frac{\varepsilon}{2} \quad \text { for all } t \geq \tau_{2} .
$$

Let $t \geq \tau_{2}$ and $x \in \varphi(-t, p) C$. If $\min \left\{\left|x-\varphi(-t, p) c_{1}\right|,\left|x-\varphi(-t, p) c_{2}\right|\right\} \leq \frac{\varepsilon}{2}$, then (17.2) implies that $d\left(x, \hat{M}_{i}\left(\theta_{-t} p\right)\right) \leq \varepsilon$. Otherwise, since $x \in \bar{R}_{i-1}\left(\theta_{-t} p\right)$ and due to (17.3), there exists a $y \in \hat{R}_{i-1}\left(\theta_{-t} p\right)$ with $|x-y| \leq \frac{\varepsilon}{2}$. Obviously,

$$
y \in \varphi(-t, p) C \subset \varphi(-t, p) \bar{M}_{i}(p) \subset R_{i}^{*}\left(\theta_{-t} p\right)
$$

is fulfilled. Hence, $y \in \hat{M}_{i}\left(\theta_{-t} p\right)$ and thus, $d\left(x, \hat{M}_{i}\left(\theta_{-t} p\right)\right) \leq \varepsilon$. This finishes the proof of this step.

Step 3. The relation

$$
\lim _{t \rightarrow \infty} d\left(M_{i}\left(\theta_{-t} p\right), \hat{M}_{i}\left(\theta_{-t} p\right)\right)=0
$$

is fulfilled.

Since $\bar{M}_{i}(p)$ has only finitely many connected components, this assertion follows from Step 2 and the fact that $\bar{M}_{i} \supset M_{i}$.

\section{Past Morse decompositions of Linear systems}

In this section, we study past Morse decompositions of linear nonautonomous dynamical systems. Under the assumption that the base space is chain recurrent, such (autonomous) Morse decompositions of the corresponding skew product flow have been studied in Selgrade [20, Salamon and Zehnder [19] and Colonius and Kliemann [6, Chapter 5] (see also Colonius and Kliemann [5, 7] and Braga BARROS and SAN MARTIN [4]). 
For $N \in \mathbb{N}$, the Euclidean space $\mathbb{R}^{N}$ is equipped with the Euclidean norm $\|\cdot\|$, which is induced by the scalar product $\langle\cdot, \cdot\rangle$, defined by $\langle x, y\rangle:=\sum_{i=1}^{N} x_{i} y_{i}$, where $x=\left(x_{1}, \ldots, x_{N}\right), y=\left(y_{1}, \ldots, y_{N}\right) \in \mathbb{R}^{N}$. To introduce the real projective space $\mathbb{P}^{N-1}$ of the $\mathbb{R}^{N}$, we say that two elements $x, y \in \mathbb{R}^{N} \backslash\{0\}$ are equivalent if there exists a $c \in \mathbb{R}$ such that $x=c y$. The equivalence class of $x \in \mathbb{R}^{N}$ is denoted by $\mathbb{P} x$, and we call the set of all equivalence classes the projective space $\mathbb{P}^{N-1}$. Equipped with the metric $d_{\mathbb{P}}: \mathbb{P}^{N-1} \times \mathbb{P}^{N-1} \rightarrow[0, \sqrt{2}]$, given by

$$
d_{\mathbb{P}}(\mathbb{P} v, \mathbb{P} w)=\min \left\{\left\|\frac{v}{\|v\|}-\frac{w}{\|w\|}\right\|,\left\|\frac{v}{\|v\|}+\frac{w}{\|w\|}\right\|\right\} \quad \text { for all } v, w \in \mathbb{R}^{N},
$$

the projective space is a compact metric space. For any $v \in \mathbb{P}^{N-1}$, we define $\mathbb{P}^{-1} v:=\left\{x \in \mathbb{R}^{N}: \mathbb{P} x=v\right\} \cup\{0\}$.

Before considering nonautonomous dynamical systems on the projective space, we state a fundamental lemma, which follows from Colonius and KliEmann 6 , Lemma B.1.17, p. 538].

Lemma 8.1. For all $\varepsilon>0$, there exists a $\delta \in(0,1)$ such that for all $v, w \in \mathbb{R}^{N} \backslash\{0\}$ with

we have

$$
\frac{\langle v, w\rangle^{2}}{\|v\|^{2}\|w\|^{2}} \geq 1-\delta
$$

$$
d_{\mathbb{P}}(\mathbb{P} v, \mathbb{P} w) \leq \varepsilon
$$

Let $\left(\theta: \mathbb{T} \times P \rightarrow P, \varphi: \mathbb{T} \times P \times \mathbb{R}^{N} \rightarrow \mathbb{R}^{N}\right)$ be a linear nonautonomous dynamical system, i.e., given $\alpha, \beta \in \mathbb{R}$, we have

$$
\varphi(t, p, \alpha x+\beta y)=\alpha \varphi(t, p, x)+\beta \varphi(t, p, y) \quad \text { for all } t \in \mathbb{T}, p \in P \text { and } x, y \in \mathbb{R}^{N} \text {. }
$$

Thus, there exists a matrix-valued function $\Phi: \mathbb{T} \times P \rightarrow \mathbb{R}^{N \times N}$ with $\Phi(t, p) x=$ $\varphi(t, p, x)$ for all $t \in \mathbb{T}, p \in P$ and $x \in \mathbb{R}^{N}$. The $\operatorname{NDS}(\theta, \varphi)$ canonically induces a nonautonomous dynamical system $(\theta, \mathbb{P} \Phi)$ on the $\mathbb{P}^{N-1}$ by defining

$$
\mathbb{P} \Phi(t, p) \mathbb{P} x:=\mathbb{P}(\Phi(t, p) x) \quad \text { for all } \tau, t \in \mathbb{T}, p \in P \text { and } x \in \mathbb{R}^{N}
$$

(see Colonius and Kliemann [6, Lemma 5.2.1, p. 149]).

The main observation of the following proposition is that past attractors in $\mathbb{P}^{N-1}$ give rise to linear subspaces in $\mathbb{R}^{N}$. For a similar result, see SALAMON and ZEHNDER [19, Proposition 2.9] and Colonius and Kliemann [6, Lemma 5.2.2, p. 149].

Proposition 8.2 (Past attractors in $\left.\mathbb{P}^{N-1}\right)$. Let $A$ be a past attractor of $(\theta, \mathbb{P} \Phi)$. Then, for all $p \in P$ and all compact sets $C \subset \mathbb{S}^{N-1} \backslash \mathbb{P}^{-1} A(p)$, we have

$$
\lim _{t \rightarrow \infty} \frac{\sup _{v \in \mathbb{S}^{N-1} \cap \mathbb{P}^{-1} A(p)}\|\Phi(-t, p) v\|}{\inf _{w \in C}\|\Phi(-t, p) w\|}=0 .
$$

Moreover, for all $p \in P$, the set $\mathbb{P}^{-1} A(p)$ is a linear subspace of $\mathbb{R}^{N}$.

Proof. Let $A$ be a past attractor of $(\theta, \mathbb{P} \Phi)$, and choose $p \in P$ and a compact set $C \subset \mathbb{S}^{N-1} \backslash \mathbb{P}^{-1} A(p)$ arbitrarily. First, we define for any nonzero $v \in \mathbb{P}^{-1} A(p)$ and $w \in C$ the two-dimensional linear subspace $L_{v, w} \subset \mathbb{R}^{N}$ by

$$
L_{v, w}:=\{r v+s w: r, s \in \mathbb{R}\} .
$$

The proof of this proposition is divided into five steps. 
Step 1. For all nonzero $v \in \mathbb{P}^{-1} A(p)$ and $w \in C$ such that $\mathbb{P} v$ is a boundary point of $A(p) \cap \mathbb{P} L_{v, w}$ relative to $\mathbb{P} L_{v, w}$, we have

$$
\lim _{t \rightarrow \infty} \frac{\|\Phi(-t, p) v\|}{\|\Phi(-t, p) w\|}=0 .
$$

Since $A$ is a past attractor, there exists an $\eta>0$ such that

$$
\lim _{t \rightarrow \infty} d\left(\mathbb{P} \Phi\left(t, \theta_{-t} p\right) U_{2 \eta}\left(A\left(\theta_{-t} p\right)\right) \mid A(p)\right)=0 .
$$

Due to Lemma 8.1 there exists a $\delta \in(0,1)$ such that for all $u_{1}, u_{2} \in \mathbb{R}^{N} \backslash\{0\}$ with

$$
\frac{\left\langle u_{1}, u_{2}\right\rangle^{2}}{\left\|u_{1}\right\|^{2}\left\|u_{2}\right\|^{2}} \geq 1-\delta,
$$

we have $d_{\mathbb{P}}\left(\mathbb{P} u_{1}, \mathbb{P} u_{2}\right) \leq \eta$. We argue negatively and suppose that there exist a $\gamma>0$ and a sequence $\left\{t_{n}\right\}_{n \in \mathbb{N}}$ with $\lim _{n \rightarrow \infty} t_{n}=-\infty$ such that

$$
\frac{\left\|\Phi\left(t_{n}, p\right) w\right\|}{\left\|\Phi\left(t_{n}, p\right) v\right\|} \leq \gamma \quad \text { for all } n \in \mathbb{N} .
$$

For nonzero $c \in \mathbb{R}$ with $|c|$ sufficiently small, this implies that for all $n \in \mathbb{N}$,

$$
\begin{aligned}
& \frac{\left\langle\Phi\left(t_{n}, p\right)(c w+v), \Phi\left(t_{n}, p\right) v\right\rangle^{2}}{\left\|\Phi\left(t_{n}, p\right)(c w+v)\right\|^{2}\left\|\Phi\left(t_{n}, p\right) v\right\|^{2}} \\
= & \frac{c^{2}\left\langle\Phi\left(t_{n}, p\right) w, \Phi\left(t_{n}, p\right) v\right\rangle^{2}+2 c\left\|\Phi\left(t_{n}, p\right) v\right\|^{2}\left\langle\Phi\left(t_{n}, p\right) w, \Phi\left(t_{n}, p\right) v\right\rangle+\left\|\Phi\left(t_{n}, p\right) v\right\|^{4}}{c^{2}\left\|\Phi\left(t_{n}, p\right) w\right\|^{2}\left\|\Phi\left(t_{n}, p\right) v\right\|^{2}+2 c\left\|\Phi\left(t_{n}, p\right) v\right\|^{2}\left\langle\Phi\left(t_{n}, p\right) w, \Phi\left(t_{n}, p\right) v\right\rangle+\left\|\Phi\left(t_{n}, p\right) v\right\|^{4}} \\
\geq & 1-\delta
\end{aligned}
$$

holds. Hence, for $|c|>0$ sufficiently small, we have

$$
d_{\mathbb{P}}\left(\mathbb{P} \Phi\left(t_{n}, p\right) \mathbb{P}(c w+v), A\left(\theta_{t_{n}} p\right)\right) \leq \eta \quad \text { for all } n \in \mathbb{N} .
$$

This implies that

$$
\begin{aligned}
d_{\mathbb{P}}(\mathbb{P}(c w+v), A(p)) & =\lim _{n \rightarrow \infty} d_{\mathbb{P}}(\mathbb{P}(c w+v), A(p)) \\
& =\lim _{n \rightarrow \infty} d_{\mathbb{P}}(\mathbb{P} \Phi\left(-t_{n}, \theta_{t_{n}} p\right) \underbrace{\mathbb{P} \Phi\left(t_{n}, p\right) \mathbb{P}(c w+v)}_{\in U_{2 \eta}\left(A\left(\theta_{t_{n}} p\right)\right)}, A(p)) \\
& \stackrel{\text { 8.1 }}{=} 0 .
\end{aligned}
$$

This is a contradiction, since $\mathbb{P} v$ is assumed to be a boundary point of $A(p) \cap \mathbb{P} L_{v, w}$ in $\mathbb{P} L_{v, w}$, and thus, the first step of this proof is finished.

Step 2. For all nonzero $v \in \mathbb{P}^{-1} A(p)$ and $w \in C$, the set $A(p) \cap \mathbb{P} L_{v, w}$ is a singleton.

For this purpose, we note that any point in $\mathbb{P} L_{v, w} \backslash\{\mathbb{P} v\}$ is given by $\mathbb{P}(w+c v)$ for some $c \in \mathbb{R}$. It follows from Step 1 that

$$
\begin{aligned}
& \lim _{t \rightarrow-\infty} \frac{\langle\Phi(t, p)(w+c v), \Phi(t, p) w\rangle^{2}}{\|\Phi(t, p)(w+c v)\|^{2}\|\Phi(t, p) w\|^{2}} \\
= & \lim _{t \rightarrow-\infty} \frac{\|\Phi(t, p) w\|^{4}+2 c\|\Phi(t, p) w\|^{2}\langle\Phi(t, p) v, \Phi(t, p) w\rangle+c^{2}\langle\Phi(t, p) v, \Phi(t, p) w\rangle^{2}}{\|\Phi(t, p) w\|^{4}+2 c\|\Phi(t, p) w\|^{2}\langle\Phi(t, p) v, \Phi(t, p) w\rangle+c^{2}\|\Phi(t, p) v\|^{2}\|\Phi(t, p) w\|^{2}}=1
\end{aligned}
$$

in case $\mathbb{P} v$ is a boundary point of $A(p) \cap \mathbb{P} L_{v, w}$ relative to $\mathbb{P} L_{v, w}$. This implies with Lemma 8.1 that

$$
\lim _{t \rightarrow-\infty} d_{\mathbb{P}}(\mathbb{P} \Phi(t, p) \mathbb{P}(w+c v), \mathbb{P} \Phi(t, p) \mathbb{P} w)=0,
$$

and hence, $\mathbb{P}(w+c v) \notin A(p)$. Therefore, $A(p) \cap \mathbb{P} L_{v, w}$ consists of a single point. 
Step 3. For all nonzero $v \in \mathbb{P}^{-1} A(p)$ and $w \in C$, we have

$$
\lim _{t \rightarrow \infty} \frac{\|\Phi(-t, p) v\|}{\|\Phi(-t, p) w\|}=0
$$

This follows directly from Step 1 and Step 2 .

Step 4. $\mathbb{P}^{-1} A(p)$ is a linear subspace of $\mathbb{R}^{N}$.

We have shown that for any two-dimensional subspace $L_{v, w}$, the set $A(p) \cap \mathbb{P} L_{v, w}$ is either empty, equals $\mathbb{P} L_{v, w}$ or consists of a single point. This implies that $\mathbb{P}^{-1} A$ intersects each fiber in a linear subspace.

Step 5. We have

$$
\lim _{t \rightarrow \infty} \frac{\sup _{v \in \mathbb{S}^{N-1} \cap \mathbb{P}^{-1} A(p)}\|\Phi(-t, p) v\|}{\inf _{w \in C}\|\Phi(-t, p) w\|}=0
$$

We assume to the contrary that there exist sequences $\left\{t_{n}\right\}_{n \in \mathbb{N}}$ in $\mathbb{R},\left\{v_{n}\right\}_{n \in \mathbb{N}}$ in $\mathbb{S}^{N-1} \cap \mathbb{P}^{-1} A(p)$ and $\left\{w_{n}\right\}_{n \in \mathbb{N}}$ in $C$ such that $\lim _{n \rightarrow \infty} t_{n}=-\infty$ and, w.l.o.g., $\lim _{n \rightarrow \infty} v_{n}=v$ and $\lim _{n \rightarrow \infty} w_{n}=w$ for some $v \in \mathbb{P}^{-1} A(p) \cap \mathbb{S}^{N-1}$ and $w \in C$, and the following property is fulfilled: There exists a $\gamma>0$ such that

$$
\frac{\left\|\Phi\left(t_{n}, p\right) w_{n}\right\|}{\left\|\Phi\left(t_{n}, p\right) v_{n}\right\|} \leq \gamma \quad \text { for all } n \in \mathbb{N} .
$$

Similarly to Step 1 , for nonzero $c \in \mathbb{R}$ with $|c|$ sufficiently small, this implies that for all $n \in \mathbb{N}$,

$$
\begin{aligned}
& \frac{\left\langle\Phi\left(t_{n}, p\right)\left(c w_{n}+v_{n}\right), \Phi\left(t_{n}, p\right) v_{n}\right\rangle^{2}}{\left\|\Phi\left(t_{n}, p\right)\left(c w_{n}+v_{n}\right)\right\|^{2}\left\|\Phi\left(t_{n}, p\right) v_{n}\right\|^{2}} \\
= & \frac{c^{2}\left\langle\Phi\left(t_{n}, p\right) w_{n}, \Phi\left(t_{n}, p\right) v_{n}\right\rangle^{2}+2 c\left\|\Phi\left(t_{n}, p\right) v_{n}\right\|^{2}\left\langle\Phi\left(t_{n}, p\right) w_{n}, \Phi\left(t_{n}, p\right) v_{n}\right\rangle+\left\|\Phi\left(t_{n}, p\right) v_{n}\right\|^{4}}{c^{2}\left\|\Phi\left(t_{n}, p\right) w_{n}\right\|^{2}\left\|\Phi\left(t_{n}, p\right) v_{n}\right\|^{2}+2 c\left\|\Phi\left(t_{n}, p\right) v_{n}\right\|^{2}\left\langle\Phi\left(t_{n}, p\right) w_{n}, \Phi\left(t_{n}, p\right) v_{n}\right\rangle+\left\|\Phi\left(t_{n}, p\right) v_{n}\right\|^{4}} \\
\geq & 1-\delta
\end{aligned}
$$

holds, with $\delta \in(0,1)$ chosen as in Step 1 . Hence, for $|c|>0$ sufficiently small, we have

$$
d_{\mathbb{P}}\left(\mathbb{P} \Phi\left(t_{n}, p\right) \mathbb{P}\left(c w_{n}+v_{n}\right), A\left(\theta_{t_{n}} p\right)\right) \leq \eta \quad \text { for all } n \in \mathbb{N} .
$$

Since $\mathbb{P}(c w+v) \notin A(p)$ (due to Step 2, $A(p) \cap \mathbb{P} L_{v, w}$ is a singleton), there exist an $n_{0} \in \mathbb{N}$ and a $\beta>0$ such that $c w_{n}+v_{n} \notin \mathbb{P}^{-1} U_{\beta}(A(p))$ for all $n \geq n_{0}$. Similarly to Step 1, using (8.1), this implies a contradiction.

Concerning past repellers, we cannot expect that their fibers give rise to linear subspaces, since they are intrinsically nonunique (see Remark 3.2). The following proposition, however, says that for any past attractor, we easily can find a linear counterpart in the form of a past repeller.

Proposition 8.3 (Past repellers in $\left.\mathbb{P}^{N-1}\right)$. Let $A$ be a past attractor of $(\theta, \mathbb{P} \Phi)$ and $R \subset P \times \mathbb{P}^{N-1}$ be an invariant nonautonomous set such that $\mathbb{P}^{-1} R(p)$ is a linear subspace of the $\mathbb{R}^{N}$ and

$$
\mathbb{P}^{-1} A(p) \oplus \mathbb{P}^{-1} R(p)=\mathbb{R}^{N} \quad \text { for all } p \in P .
$$

Then, $R$ is a past repeller, and the relation $A=R^{*}$ is fulfilled. 
Proof. The proof of this proposition is divided into five steps.

Step 1. For all $p \in P$ and compact sets $C \subset \mathbb{P}^{N-1}$ with $C \cap A(p)=\emptyset$, we have

$$
\lim _{t \rightarrow \infty} \inf _{0 \neq v \in \mathbb{P}^{-1} C} \frac{\left\|\Phi(-t, p) v_{r}\right\|}{\|\Phi(-t, p) v\|}=\lim _{t \rightarrow \infty} \sup _{0 \neq v \in \mathbb{P}^{-1} C} \frac{\left\|\Phi(-t, p) v_{r}\right\|}{\|\Phi(-t, p) v\|}=1,
$$

where $v=v_{a}+v_{r}$ with $v_{a} \in \mathbb{P}^{-1} A(p)$ and $v_{r} \in \mathbb{P}^{-1} R(p)$.

The first assertion follows from

$$
\begin{aligned}
& \lim _{t \rightarrow \infty} \inf _{0 \neq v \in \mathbb{P}^{-1} C} \frac{\left\|\Phi(-t, p) v_{r}\right\|}{\|\Phi(-t, p) v\|} \\
\geq \quad & \left(\lim _{t \rightarrow \infty} \sup _{0 \neq v \in \mathbb{P}^{-1} C} \frac{\left\|\Phi(-t, p) v_{a}\right\|}{\left\|\Phi(-t, p) v_{r}\right\|}+1\right)^{-1} \\
= & \left(\lim _{t \rightarrow \infty} \sup _{v \in \mathbb{P}^{-1} C, v_{a} \neq 0} \frac{\left\|v_{a}\right\|\left\|\Phi(-t, p) \frac{v_{a}}{\left\|v_{a}\right\|}\right\|}{\left\|v_{r}\right\|\left\|\Phi(-t, p) \frac{v_{r}}{\left\|v_{r}\right\|}\right\|}+1\right)^{-1}
\end{aligned}
$$

$\stackrel{\text { Proposition } 8.2}{=} 1$

and

$$
\begin{aligned}
& \lim _{t \rightarrow \infty} \inf _{0 \neq v \in \mathbb{P}^{-1} C} \frac{\left\|\Phi(-t, p) v_{r}\right\|}{\|\Phi(-t, p) v\|} \\
& \leq \quad\left(\lim _{t \rightarrow \infty} \sup _{0 \neq v \in \mathbb{P}^{-1} C}\left|1-\frac{\left\|\Phi(-t, p) v_{a}\right\|}{\left\|\Phi(-t, p) v_{r}\right\|}\right|\right)^{-1} \\
& =\quad\left(\lim _{t \rightarrow \infty} \sup _{v \in \mathbb{P}^{-1} C, v_{a} \neq 0}\left|1-\frac{\left\|v_{a}\right\|\left\|\Phi(-t, p) \frac{v_{a}}{\left\|v_{a}\right\|}\right\|}{\left\|v_{r}\right\|\left\|\Phi(-t, p) \frac{v_{r}}{\left\|v_{r}\right\|}\right\|}\right|\right)^{-1} \\
& \text { Proposition } \stackrel{8.2}{=} 1 \text {. }
\end{aligned}
$$

In both relations, Proposition 8.2 is applicable, because the set $\left\{v_{a}: v \in \mathbb{P}^{-1} C \cap\right.$ $\left.\mathbb{S}^{N-1}\right\}$ is compact and the set $\left\{v_{r}: v \in \mathbb{P}^{-1} C \cap \mathbb{S}^{N-1}\right\}$ is bounded away from zero. This is due to the fact that the projector $Q \in \mathbb{R}^{N \times N}$ with range $\mathbb{P}^{-1} A(p)$ and null space $\mathbb{P}^{-1} R(p)$ satisfies

$$
\left\{v_{a}: v \in \mathbb{P}^{-1} C \cap \mathbb{S}^{N-1}\right\}=Q\left(\mathbb{P}^{-1} C \cap \mathbb{S}^{N-1}\right)
$$

and

$$
\left\{v_{r}: v \in \mathbb{P}^{-1} C \cap \mathbb{S}^{N-1}\right\}=(i d-Q)\left(\mathbb{P}^{-1} C \cap \mathbb{S}^{N-1}\right) .
$$

The assertion

$$
\lim _{t \rightarrow \infty} \sup _{0 \neq v \in \mathbb{P}^{-1} C} \frac{\left\|\Phi(-t, p) v_{r}\right\|}{\|\Phi(-t, p) v\|}=1
$$

follows analogously.

Step 2. For all $p \in P$ and compact sets $C \subset \mathbb{P}^{N-1}$ with $C \cap A(p)=\emptyset$, we have

$$
\lim _{t \rightarrow \infty} d_{\mathbb{P}}\left(\mathbb{P} \Phi(-t, p) C \mid R\left(\theta_{-t} p\right)\right)=0 .
$$


With $v_{a}$ and $v_{r}$ defined as in Step 1 , for all $t \geq 0$ and $v \in \mathbb{S}^{N-1} \cap \mathbb{P}^{-1} C$, we consider the expression

$$
\begin{aligned}
& \frac{\left\langle\Phi(-t, p) v, \Phi(-t, p) v_{r}\right\rangle^{2}}{\|\Phi(-t, p) v\|^{2}\left\|\Phi(-t, p) v_{r}\right\|^{2}} \\
= & \frac{\left(\left\langle\Phi(-t, p) v_{a}, \Phi(-t, p) v_{r}\right\rangle+\left\langle\Phi(-t, p) v_{r}, \Phi(-t, p) v_{r}\right\rangle\right)^{2}}{\|\Phi(-t, p) v\|^{2}\left\|\Phi(-t, p) v_{r}\right\|^{2}} \\
= & \frac{\left\langle\Phi(-t, p) v_{a}, \Phi(-t, p) v_{r}\right\rangle^{2}}{\|\Phi(-t, p) v\|^{2}\left\|\Phi(-t, p) v_{r}\right\|^{2}}+\frac{\left\|\Phi(-t, p) v_{r}\right\|^{2}}{\|\Phi(-t, p) v\|^{2}}+\frac{2\left\langle\Phi(-t, p) v_{a}, \Phi(-t, p) v_{r}\right\rangle}{\|\Phi(-t, p) v\|^{2}} .
\end{aligned}
$$

Using the Cauchy-Schwarz inequality, we obtain the following relations:

$$
\begin{aligned}
0 \leq \lim _{t \rightarrow \infty} \sup _{v \in \mathbb{S}^{N-1} \cap \mathbb{P}^{-1} C} \frac{\left\langle\Phi(-t, p) v_{a}, \Phi(-t, p) v_{r}\right\rangle^{2}}{\|\Phi(-t, p) v\|^{2}\left\|\Phi(-t, p) v_{r}\right\|^{2}} \\
\quad \leq \lim _{t \rightarrow \infty} \sup _{v \in \mathbb{S}^{N-1} \cap \mathbb{P}^{-1} C} \frac{\left\|\Phi(-t, p) v_{a}\right\|^{2}}{\|\Phi(-t, p) v\|^{2}} \stackrel{\text { Proposition 8.2 }}{=} 0
\end{aligned}
$$

and

$$
\begin{aligned}
& 0 \leq \quad \lim _{t \rightarrow \infty} \sup _{v \in \mathbb{S}^{N-1} \cap \mathbb{P}^{-1} C} \frac{2\left|\left\langle\Phi(-t, p) v_{a}, \Phi(-t, p) v_{r}\right\rangle\right|}{\|\Phi(-t, p) v\|^{2}} \\
& \leq \lim _{t \rightarrow \infty} \sup _{v \in \mathbb{S}^{N-1} \cap \mathbb{P}^{-1} C} 2 \frac{\left\|\Phi(-t, p) v_{a}\right\|}{\|\Phi(-t, p) v\|} \frac{\left\|(-t, p) v_{r}\right\|}{\|\Phi(-t, p) v\|} \\
& \stackrel{\text { Step }}{=} \lim _{t \rightarrow \infty} \sup _{v \in \mathbb{S}^{N-1} \cap \mathbb{P}^{-1} C} \frac{2\left\|\Phi(-t, p) v_{a}\right\|}{\|\Phi(-t, p) v\|} \\
& \stackrel{\text { Proposition 8.2 }}{=} 0 .
\end{aligned}
$$

Hence, we obtain

$$
\begin{gathered}
\lim _{t \rightarrow \infty} \inf _{v \in \mathbb{S}^{N-1} \cap \mathbb{P}^{-1} C} \frac{\left\langle\Phi(-t, p) v, \Phi(-t, p) v_{r}\right\rangle^{2}}{\|\Phi(-t, p) v\|^{2}\left\|\Phi(-t, p) v_{r}\right\|^{2}} \\
=\lim _{t \rightarrow \infty} \inf _{v \in \mathbb{S}^{N}-1 \cap \mathbb{P}^{-1} C}\left(\frac{\left\langle\Phi(-t, p) v_{a}, \Phi(-t, p) v_{r}\right\rangle^{2}}{\|\Phi(-t, p) v\|^{2}\left\|\Phi(-t, p) v_{r}\right\|^{2}}+\frac{\left\|\Phi(-t, p) v_{r}\right\|^{2}}{\|\Phi(-t, p) v\|^{2}}\right. \\
\left.+\frac{2\left\langle\Phi(-t, p) v_{a}, \Phi(-t, p) v_{r}\right\rangle}{\|\Phi(-t, p) v\|^{2}}\right)
\end{gathered}
$$

Step ${ }^{1} 1$

Using Lemma 8.1, this implies the assertion.

Step 3. A and $R$ are past isolated, i.e., there exists a $\beta>0$ such that for all $p \in P$, there exists a $\tau>0$ with

$$
U_{\beta}\left(A\left(\theta_{-t} p\right)\right) \cap U_{\beta}\left(R\left(\theta_{-t} p\right)\right)=\emptyset \quad \text { for all } t \geq \tau .
$$

Since $A$ is a past attractor, there exists an $\eta>0$ such that for all $p \in P$, we have

$$
\lim _{t \rightarrow \infty} d_{\mathbb{P}}\left(\mathbb{P} \Phi\left(t, \theta_{-t} p\right) U_{\eta}\left(A\left(\theta_{-t} p\right)\right) \mid A(p)\right)=0 .
$$

Defining $\beta:=\frac{\eta}{2}$ and using the invariance of $R$, this implies the assertion. 
Step 4. $R$ is a past repeller.

This is a direct consequence of Step 2 and Step 3.

Step 5. The relation $A=R^{*}$ is fulfilled.

We define $\eta>0, P^{*}$ and $B_{\zeta}$ for $\zeta \in(0, \eta]$ as in the preamble of Theorem 4.3 . We also consider the collection $\mathcal{D}:=\left\{B_{\zeta}: \zeta \in(0, \eta]\right\}$. Due to Theorem 4.3, it is sufficient to show that $A$ is a $\mathcal{D}$-past attractor. Thereto, we fix $\zeta \in(0, \eta]$ and $p \in P^{*}$. Furthermore, we choose $\varepsilon>0$ arbitrarily and consider the compact set $C:=\mathbb{P}^{N-1} \backslash U_{\varepsilon}(A(p))$. Due to Step 2, we have

$$
\lim _{t \rightarrow \infty} d\left(\mathbb{P} \Phi(-t, p) C \mid R\left(\theta_{-t} p\right)\right)=0 .
$$

This implies that there exists a $\tau>0$ such that $\mathbb{P} \Phi(-t, p) C \cap B_{\zeta}\left(\theta_{-t} p\right)=\emptyset$ for all $t \geq \tau$. Thus,

$$
d_{\mathbb{P}}\left(\mathbb{P} \Phi\left(t, \theta_{-t} p\right) B_{\zeta}\left(\theta_{-t} p\right) \mid A(p)\right) \leq \varepsilon \quad \text { for all } t \geq \tau
$$

i.e., $A$ is a $\mathcal{D}$-past attractor. This finishes the proof of this proposition.

Lemma 8.4. For $n \in \mathbb{N}$, consider nontrivial linear subspaces $W_{n} \subsetneq V_{n} \subsetneq \mathbb{R}^{N}$. Furthermore, let $\left\{x_{n}\right\}_{n \in \mathbb{N}}$ be a sequence in $\mathbb{R}^{N}$ such that the following hypotheses are fulfilled:

(i) $x_{n} \notin V_{n}$ for all $n \in \mathbb{N}$,

(ii) $\lim _{n \rightarrow \infty} d_{\mathbb{P}}\left(\mathbb{P} x_{n}, \mathbb{P} V_{n}\right)=0$,

(iii) there exists an $\varepsilon>0$ such that $d_{\mathbb{P}}\left(\mathbb{P} x_{n}, \mathbb{P} W_{n}\right) \geq \varepsilon$ for all $n \in \mathbb{N}$.

For all $n \in \mathbb{N}$, we define $C_{n}:=W_{n} \oplus\left\{\lambda x_{n}: \lambda \in \mathbb{R}\right\}$. Then, the limit relation

$$
\lim _{n \rightarrow \infty} d_{\mathbb{P}}\left(\mathbb{P} C_{n} \mid \mathbb{P} V_{n}\right)=0
$$

is fulfilled.

Proof. W.l.o.g., we assume that $\left\|x_{n}\right\|=1$ for all $n \in \mathbb{N}$. Due to hypothesis (ii), there exists a sequence $\left\{v_{n}\right\}_{n \in \mathbb{N}}$ with $v_{n} \in V_{n}$ and $\left\|v_{n}\right\|=1$ for all $n \in \mathbb{N}$ such that $\lim _{n \rightarrow \infty}\left\|x_{n}-v_{n}\right\|=0$. Since $\mathbb{P} C_{n}$ is a compact subset of $\mathbb{P}^{N-1}$, there exists a sequence $\left\{c_{n}\right\}_{n \in \mathbb{N}}$ with $c_{n} \in C_{n}$ for all $n \in \mathbb{N}$ such that $d_{\mathbb{P}}\left(\mathbb{P} C_{n} \mid \mathbb{P} V_{n}\right)=d_{\mathbb{P}}\left(\mathbb{P} c_{n}, \mathbb{P} V_{n}\right)$. W.l.o.g., we assume that $c_{n}$ is of the form

$$
c_{n}:=x_{n}+w_{n} \text { for all } n \in \mathbb{N} \text {, }
$$

where $\left\{w_{n}\right\}_{n \in \mathbb{N}}$ is a sequence with $w_{n} \in W_{n}$ for all $n \in \mathbb{N}$, and we define

$$
r_{n}:=v_{n}+w_{n} \quad \text { for all } n \in \mathbb{N}
$$

and $\beta_{n}:=\left\langle x_{n}, w_{n}\right\rangle, \delta_{n}:=\left\langle v_{n}, w_{n}\right\rangle$ and $\gamma_{n}:=\left\langle x_{n}, v_{n}\right\rangle$ for all $n \in \mathbb{N}$. Then, for all $n \in \mathbb{N}$, we have

$$
\begin{aligned}
& \frac{\left\langle c_{n}, r_{n}\right\rangle^{2}}{\left\|c_{n}\right\|^{2}\left\|r_{n}\right\|^{2}} \\
= & \frac{\gamma_{n}^{2}+\overbrace{\beta_{n}^{2}+\delta_{n}^{2}+\left\|w_{n}\right\|^{4}+2 \gamma_{n}\left\|x_{n}\right\|^{2}+2 \beta_{n} \delta_{n}}^{\delta_{n}}+\overbrace{2\left(\beta_{n}+\delta_{n}\right)\left(\left\|w_{n}\right\|^{2}+\gamma_{n}\right)}^{2\left(\left\|w_{n}\right\|^{2}+\left\|w_{n}\right\|^{4}+4 \beta_{n} \delta_{n}\right.}+\underbrace{2\left(\beta_{n}+\delta_{n}\right)\left(\left\|w_{n}\right\|^{2}+1\right)}_{=: \tilde{\xi}_{n}}}{1+\underbrace{2\left(\beta_{n}\right.}_{\tilde{\eta}_{n}}},
\end{aligned}
$$


and it is easy to see that $\lim _{n \rightarrow \infty} \gamma_{n}=1, \lim _{n \rightarrow \infty} \frac{\xi_{n}}{\tilde{\xi}_{n}}=1$ and $\lim _{n \rightarrow \infty} \frac{\eta_{n}}{\tilde{\eta}_{n}}=1$. This implies that

$$
\lim _{n \rightarrow \infty} \frac{\left\langle c_{n}, r_{n}\right\rangle^{2}}{\left\|c_{n}\right\|^{2}\left\|r_{n}\right\|^{2}}=1
$$

in case $\left\|c_{n}\right\|\left\|r_{n}\right\|$ is bounded away from 0 in the limit $n \rightarrow \infty$. To see that this is fulfilled, we need hypothesis (iii), which yields that there exists a $\delta \in(0,1)$ with

$$
\frac{\left\langle x_{n}, w\right\rangle}{\|w\|} \leq \delta \quad \text { for all } n \in \mathbb{N} \text { and } w \in W_{n}
$$

(see Lemma 8.1). This means that for all $n \in \mathbb{N}$ and $w \in W_{n}$, we have

$$
\left\|x_{n}-w\right\|^{2}=1-2\left\langle x_{n}, w\right\rangle+\|w\|^{2} \geq 1-2 \delta\|w\|+\|w\|^{2} \geq \gamma \quad \text { for some } \gamma>0,
$$

and this finishes the proof of this lemma.

In our special situation, convergence in both directions to the Morse sets is satisfied.

Theorem 8.5 (Dynamical properties of past Morse decompositions of linear systems). Let $\left\{M_{1}, \ldots, M_{n}\right\}$ be a past Morse decomposition obtained by the finite sequence of past repellers $R_{0} \supset \cdots \supset R_{n}$ such that $\mathbb{P}^{-1} R_{i}(p)$ is a linear subspace of $\mathbb{R}^{N}$ for $i \in\{1, \ldots, n-1\}$ and $p \in P$. Then, the following statements hold:

(i) Pullback convergence. For all $p \in P$ and all functions $\gamma: \mathbb{T}^{+} \rightarrow \mathbb{P}^{N-1}$ with

$$
\liminf _{t \rightarrow \infty} d_{\mathbb{P}}\left(\gamma(t), \bigcup_{j=1, \ldots, n} \partial R_{j}\left(\theta_{-t} p\right)\right)>0,
$$

we have

$$
\lim _{t \rightarrow \infty} d_{\mathbb{P}}\left(\mathbb{P} \Phi\left(t, \theta_{-t} p\right) \gamma(t), \bigcup_{j=1, \ldots, n} M_{j}(p)\right)=0 .
$$

(ii) Backward convergence. For all $(p, x) \in P \times \mathbb{P}^{N-1}$, there exists an $i \in$ $\{1, \ldots, n\}$ with

$$
\lim _{t \rightarrow \infty} d_{\mathbb{P}}\left(\mathbb{P} \Phi(-t, p) x, M_{i}\left(\theta_{-t} p\right)\right)=0 .
$$

Proof. (i) This assertion is also valid for general Morse decompositions and was proved in Theorem 5.6.

(ii) Choose $(p, x) \in P \times \mathbb{P}^{N-1}$ arbitrarily. Then, there exists an $i \in\{1, \ldots, n\}$ such that

$$
x \in R_{i}^{*}(p) \text { and } x \notin R_{i-1}^{*}(p) .
$$

In case $x \in R_{i-1}(p)$, the above limit relation follows, since then $x \in M_{i}(p)$ and $M_{i}$ is invariant. We therefore assume $x \notin R_{i-1}(p)$ from now on. To obtain a contradiction, we also assume that there exist an $\varepsilon>0$ and a sequence $\left\{t_{n}\right\}_{n \in \mathbb{N}}$ in $\mathbb{R}$ with $\lim _{n \rightarrow \infty} t_{n}=\infty$ such that

$$
d_{\mathbb{P}}\left(\mathbb{P} \Phi\left(-t_{n}, p\right) x, M_{i}\left(\theta_{-t_{n}} p\right)\right) \geq \varepsilon \quad \text { for all } n \in \mathbb{N} .
$$

We define $C:=\mathbb{P}^{-1} M_{i}(p) \oplus \mathbb{P}^{-1}\{x\}$. Since $\lim _{t \rightarrow \infty} d_{\mathbb{P}}\left(\mathbb{P} \Phi(-t, p) x, R_{i-1}\left(\theta_{-t} p\right)\right)=0$ (see Theorem 4.5), Lemma 8.4 implies that

$$
\lim _{n \rightarrow \infty} d_{\mathbb{P}}\left(\mathbb{P} \Phi\left(-t_{n}, p\right) \mathbb{P} C \mid R_{i-1}\left(\theta_{-t_{n}} p\right)\right)=0 .
$$


We define $\bar{C}:=\mathbb{P}^{-1} R_{i-1}(p) \oplus \mathbb{P}^{-1}\{x\}$. Then,

$$
\begin{aligned}
\operatorname{dim}\left(\bar{C} \cap \mathbb{P}^{-1} R_{i-1}^{*}(p)\right) & =\operatorname{dim} \bar{C}+\operatorname{dim} \mathbb{P}^{-1} R_{i-1}^{*}(p)-\operatorname{dim}\left(\bar{C}+\mathbb{P}^{-1} R_{i-1}^{*}(p)\right) \\
& =N+1-N=1 .
\end{aligned}
$$

Let $y=v+w$ be a nonzero element of $\bar{C} \cap \mathbb{P}^{-1} R_{i-1}^{*}(p)$ with $v \in \mathbb{P}^{-1}\{x\}$ and $w \in \mathbb{P}^{-1} R_{i-1}(p)$. Since $y$ and $v$ are in $\mathbb{P}^{-1} R_{i}^{*}(p), w$ is also an element of $\mathbb{P}^{-1} R_{i}^{*}(p)$. Hence, $w \in \mathbb{P}^{-1} M_{i}(p)$. This implies that $y \in C$, and hence, from (8.2), we get

$$
\lim _{n \rightarrow \infty} d_{\mathbb{P}}\left(\mathbb{P} \Phi\left(-t_{n}, p\right) \mathbb{P} y \mid R_{i-1}\left(\theta_{-t_{n}} p\right)\right)=0 .
$$

This is a contradiction, since $\mathbb{P} y \in R_{i-1}^{*}(p)$ and $R_{i-1}$ and $R_{i-1}^{*}$ are isolated in the past (cf. Theorem 4.5).

In our special situation, we can generalize Proposition 4.6 .

Proposition 8.6 (Form of nonuniqueness of the Morse sets). Let $\left\{M_{1}, \ldots, M_{n}\right\}$ and $\left\{\hat{M}_{1}, \ldots, \hat{M}_{n}\right\}$ be past Morse decompositions obtained by the finite sequences of past repellers $R_{0} \supset \cdots \supset R_{n}$ and $\hat{R}_{0} \supset \cdots \supset \hat{R}_{n}$ such that $\mathbb{P}^{-1} R_{i}(p)$ and $\mathbb{P}^{-1} \hat{R}_{i}(p)$ are linear subspaces of $\mathbb{R}^{N}$ for $i \in\{1, \ldots, n-1\}$ and $p \in P$. We assume that

$$
R_{i}^{*}=\hat{R}_{i}^{*} \quad \text { for all } i \in\{1, \ldots, n-1\} .
$$

Then, the relation

$$
\lim _{t \rightarrow \infty} d_{\mathbb{P} H}\left(M_{i}\left(\theta_{-t} p\right), \hat{M}_{i}\left(\theta_{-t} p\right)\right)=0 \quad \text { for all } i \in\{1, \ldots, n\} \text { and } p \in P
$$

is fulfilled.

Proof. For $i \in\{1, n\}$, the above limit relation follows from $M_{1}=R_{1}^{*}=\hat{R}_{1}^{*}=\hat{M}_{1}$ and from Proposition 4.6, since $M_{n}=R_{n-1}$ and $\hat{M}_{n}=\hat{R}_{n-1}$. We argue negatively and assume w.l.o.g. that there exist an $i \in\{2, \ldots, n-1\}$ and a $p \in P$ such that

$$
\limsup _{t \rightarrow \infty} d_{\mathbb{P}}\left(\hat{M}_{i}\left(\theta_{-t} p\right) \mid M_{i}\left(\theta_{-t} p\right)\right)>0 .
$$

Since Proposition 4.6 implies that

$$
\lim _{t \rightarrow \infty} d_{\mathbb{P} H}\left(R_{i-1}\left(\theta_{-t} p\right), \hat{R}_{i-1}\left(\theta_{-t} p\right)\right)=0,
$$

and $\hat{R}_{i-1} \supset \hat{M}_{i}$, this means that there exist a $\gamma>0$ and sequences $\left\{t_{n}\right\}_{n \in \mathbb{N}}$ (with $\left.\lim _{n \rightarrow \infty} t_{n}=\infty\right)$ and $\left\{x_{n}\right\}_{n \in \mathbb{N}}\left(\right.$ with $\left.x_{n} \in R_{i-1}\left(\theta_{-t_{n}} p\right) \backslash M_{i}\left(\theta_{-t_{n}} p\right)\right)$ such that

$$
d_{\mathbb{P}}\left(x_{n}, M_{i}\left(\theta_{-t_{n}} p\right)\right) \geq \gamma \quad \text { for all } n \in \mathbb{N}
$$

and

$$
\lim _{n \rightarrow \infty} d_{\mathbb{P}}\left(x_{n}, \hat{M}_{i}\left(\theta_{-t_{n}} p\right)\right)=0 .
$$

The last formula implies that $\lim _{n \rightarrow \infty} d_{\mathbb{P}}\left(x_{n}, R_{i}^{*}\left(\theta_{-t_{n}} p\right)\right)=0$. We define

$$
C_{n}:=\mathbb{P}^{-1} M_{i}\left(\theta_{-t_{n}} p\right) \oplus \mathbb{P}^{-1}\left\{x_{n}\right\} \quad \text { for all } n \in \mathbb{N} .
$$

Due to Lemma 8.4, the relation

$$
\lim _{n \rightarrow \infty} d_{\mathbb{P}}\left(\mathbb{P} C_{n} \mid R_{i}^{*}\left(\theta_{-t_{n}} p\right)\right)=0
$$

holds. Since $R_{i}^{*}$ is a past attractor, we thus get

$$
\lim _{n \rightarrow \infty} d_{\mathbb{P}}\left(\mathbb{P} \Phi\left(t_{n}, \theta_{-t_{n}} p\right) \mathbb{P} C_{n} \mid R_{i}^{*}(p)\right)=0 .
$$


It is easy to see that $d_{\mathbb{P}}\left(\mathbb{P} \Phi\left(t_{n}, \theta_{-t_{n}} p\right) \mathbb{P} C_{n} \mid M_{i}(p)\right)=\sqrt{2}$ for all $n \in \mathbb{N}$, since $\mathbb{P}^{-1} \mathbb{P} \Phi\left(t_{n}, \theta_{-t_{n}} p\right) \mathbb{P} C_{n}$ has a higher dimension than $\mathbb{P}^{-1} M_{i}(p)$. This means that there exists a sequence $\left\{y_{n}\right\}_{n \in \mathbb{N}}$ with

$$
y_{n} \in \mathbb{P} \Phi\left(t_{n}, \theta_{-t_{n}} p\right) \mathbb{P} C_{n} \quad \text { and } \quad d_{\mathbb{P}}\left(y_{n}, M_{i}(p)\right) \geq 1 \quad \text { for all } n \in \mathbb{N} .
$$

Since $y_{n} \in R_{i-1}(p)$ for all $n \in \mathbb{N}$, we assume w.l.o.g. that this sequence is convergent with limit $y \in R_{i-1}(p)$. Due to (8.3), we also have $y \in R_{i}^{*}(p)$. Hence, $y \in M_{i}(p)$. This is a contradiction to (8.4).

For the rest of this article, we restrict our attention to the special situation $P=\mathbb{T}$ and $\theta(t, s)=t+s$ for all $t, s \in \mathbb{T}$. As described in Section 2, this setting includes arbitrary nonautonomous differential or difference equations. Under this assumption, we can now prove an analogon to the Theorem of Selgrade (see SELGRAdE [20, Theorem 9.7] and Colonius and Kliemann [6, Theorem 5.2.5]).

Theorem 8.7 (Finest past Morse decomposition). We suppose that $P=\mathbb{T}$ and $\theta(t, s)=t+s$ for all $t, s \in \mathbb{T}$. Then, there exists a finest past Morse decomposition $\left\{M_{1}, \ldots, M_{n}\right\}$, i.e., for any other past Morse decomposition $\left\{\tilde{M}_{1}, \ldots, \tilde{M}_{m}\right\}$, the relation

$$
\lim _{t \rightarrow \infty} d_{\mathbb{P}}\left(\bigcup_{i=1}^{n} M_{i}(-t) \mid \bigcup_{i=1}^{m} \tilde{M}_{i}(-t)\right)=0
$$

holds. Moreover, we have $n \leq N$, and the following decomposition in a Whitney sum is fulfilled:

$$
\mathbb{P}^{-1} M_{1} \oplus \cdots \oplus \mathbb{P}^{-1} M_{n}=\mathbb{T} \times \mathbb{R}^{N} .
$$

Proof. We first prove that for any two past attractors $A$ and $\bar{A}$, either the relation

$$
A \subset \bar{A} \text { or } A \supset \bar{A}
$$

is fulfilled. Supposing the contrary, due to $P=\mathbb{T}$, there exists a $\tau \in \mathbb{T}$ and elements

$$
x \in \mathbb{S}^{N-1} \cap\left(\mathbb{P}^{-1} A(\tau) \backslash \mathbb{P}^{-1} \bar{A}(\tau)\right) \quad \text { and } \quad \bar{x} \in \mathbb{S}^{N-1} \cap\left(\mathbb{P}^{-1} \bar{A}(\tau) \backslash \mathbb{P}^{-1} A(\tau)\right) .
$$

Because of Proposition 8.2, we obtain

$$
\lim _{t \rightarrow \infty} \frac{\|\Phi(-t, \tau) x\|}{\|\Phi(-t, \tau) \bar{x}\|}=0 \quad \text { and } \quad \lim _{t \rightarrow \infty} \frac{\|\Phi(-t, \tau) \bar{x}\|}{\|\Phi(-t, \tau) x\|}=0 .
$$

This is a contradiction. Proposition 8.2 also implies that the fibers of past attractors correspond to linear subspaces. Thus, there are at most $N+1$ past attractors of $(\theta, \mathbb{P} \Phi)$, namely,

$$
\emptyset=A_{0} \subsetneq A_{1} \subsetneq \cdots \subsetneq A_{n}=\mathbb{T} \times \mathbb{P}^{N-1}
$$

with $n \leq N$. Due to Proposition 8.3, it is possible to choose a sequence of past repellers $\mathbb{T} \times \mathbb{P}^{N-1}=R_{0} \supsetneq R_{1} \cdots \supsetneq R_{n}=\emptyset$ such that $R_{i}^{*}=A_{i}$ for $i \in\{0, \ldots, n\}$. We denote by $\left\{M_{1}, \ldots, M_{n}\right\}$ the corresponding past Morse decomposition. Let $\left\{\tilde{M}_{1}, \ldots, \tilde{M}_{m}\right\}$ be another past Morse decomposition, obtained by the sequence $\mathbb{T} \times \mathbb{P}^{N-1}=\tilde{R}_{0} \supsetneq \tilde{R}_{1} \supsetneq \cdots \supsetneq \tilde{R}_{m}=\emptyset$ of past repellers. Then, for each $i \in$ $\{0, \ldots, m\}$, there exists an $n_{i} \in\{0, \ldots, n\}$ such that $\tilde{R}_{i}^{*}=A_{n_{i}}$. We consider now the past Morse decomposition $\left\{\bar{M}_{1}, \ldots, \bar{M}_{n}\right\}$, which is obtained by the past repellers $R_{n_{0}}, \ldots, R_{n_{m}}$. Due to Proposition 8.6, we have

$$
\lim _{t \rightarrow \infty} d_{\mathbb{P} H}\left(\bigcup_{i=1}^{m} \bar{M}_{i}(-t), \bigcup_{i=1}^{m} \tilde{M}_{i}(-t)\right)=0 .
$$


Moreover, it is easy to see that $\bigcup_{i=1}^{n} M_{i} \subset \bigcup_{i=1}^{m} \bar{M}_{i}$ holds. This finishes the proof of the first assertion of this theorem. To show that

$$
\mathbb{P}^{-1} M_{1} \oplus \cdots \oplus \mathbb{P}^{-1} M_{n}=\mathbb{T} \times \mathbb{R}^{N},
$$

we first note that for $1 \leq i<j \leq n$, we have $\mathbb{P}^{-1} M_{i} \cap \mathbb{P}^{-1} M_{j}=\mathbb{T} \times\{0\}$ (see Proposition 5.3). Furthermore, Proposition 8.3 implies that

$$
\begin{aligned}
\mathbb{T} \times \mathbb{R}^{N} & =\mathbb{P}^{-1} R_{1}^{*}+\mathbb{P}^{-1} R_{1} \\
& =\mathbb{P}^{-1} M_{1}+\left(\mathbb{P}^{-1} R_{1} \cap\left(\mathbb{P}^{-1} R_{2}^{*}+\mathbb{P}^{-1} R_{2}\right)\right) \\
& =\mathbb{P}^{-1} M_{1}+\left(\mathbb{P}^{-1} R_{1} \cap \mathbb{P}^{-1} R_{2}^{*}\right)+\mathbb{P}^{-1} R_{2} \\
& =\mathbb{P}^{-1} M_{1}+\mathbb{P}^{-1} M_{2}+\mathbb{P}^{-1} R_{2} .
\end{aligned}
$$

Here, we used the fact that the linear subspaces $E, F, G \subset \mathbb{R}^{N}$ with $E \supset G$ fulfill

$$
E \cap(F+G)=(E \cap F)+G .
$$

It follows inductively that

$$
\mathbb{T} \times \mathbb{R}^{N}=\mathbb{P}^{-1} M_{1}+\cdots+\mathbb{P}^{-1} M_{n}+\mathbb{P}^{-1} R_{n}=\mathbb{P}^{-1} M_{1}+\cdots+\mathbb{P}^{-1} M_{n} .
$$

This finishes the proof of this theorem.

Remark 8.8. A finest past Morse decomposition $\left\{M_{1}, \ldots, M_{n}\right\}$ is not uniquely determined, but it follows directly from the above theorem that any other finest Morse decomposition $\left\{\tilde{M}_{1}, \ldots, \tilde{M}_{m}\right\}$ satisfies

$$
\lim _{t \rightarrow \infty} d_{\mathbb{P} H}\left(\bigcup_{i=1}^{n} M_{i}(-t), \bigcup_{i=1}^{m} \tilde{M}_{i}(-t)\right)=0 .
$$

Moreover, the relation $n=m$ is fulfilled.

\section{ACKNowledgement}

The author wishes to thank Professor Fritz Colonius for interesting discussions on the topic of this paper.

\section{REFERENCES}

[1] E. Akin, The General Topology of Dynamical Systems, Graduate Studies in Mathematics, no. 1, American Mathematical Society, Providence, Rhode Island, 1993. MR:1219737 (94f:58041)

[2] L. Arnold, Random Dynamical Systems, Springer, Berlin, Heidelberg, New York, 1998. MR.1723992 (2000m:37087)

[3] L. Arnold and B. Schmalfuß, Lyapunov's Second Method for Random Dynamical Systems, Journal of Differential Equations 177 (2001), no. 1, 235-265. MR.1867618(2002h:37093)

[4] C. J. Braga Barros and L. A. B. San Martin, Chain Transitive Sets for Flows on Flag Bundles, to appear in: Forum Mathematicum.

[5] F. Colonius and W. Kliemann, The Morse Spectrum of Linear Flows on Vector Bundles, Transactions of the American Mathematical Society 348 (1996), no. 11, 4355-4388. MR.1329532 (97a:58158)

[6] F. Colonius and W. Kliemann, The Dynamics of Control, Birkhäuser, 2000. MR:1752730 (2001e:93001)

[7] F. Colonius and W. Kliemann, Morse Decompositions and Spectra on Flag Bundles, Journal of Dynamics and Differential Equations 14 (2002), no. 4, 719-741. MR.1940100(2004j:37026)

[8] C. C. Conley, Isolated Invariant Sets and the Morse Index, Regional Conference Series in Mathematics, no. 38, American Mathematical Society, Providence, Rhode Island, 1978. MR.511133 (80c:58009) 
[9] H. Crauel, L. H. Duc, and S. Siegmund, Towards a Morse Theory for Random Dynamical Systems, Stochastics and Dynamics 4 (2004), no. 3, 277-296. MR2086941 (2005f:37110)

[10] F. Flandoli and B. Schmalfuß, Random Attractors for the 3-D Stochastic Navier-Stokes Equation with Mulitiplicative White Noise, Stochastics and Stochastics Reports 59 (1996), no. 1-2, 21-45. MR:1427258 (98g:60113)

[11] J. Franks, A Variation on the Poincaré-Birkhoff Theorem, Hamiltonian Dynamical Systems, Contemporary Mathematics, vol. 81, 1988, pp. 111-117. MR.986260 (90e:58095)

[12] P. E. Kloeden, Lyapunov Functions for Cocycle Attractors in Nonautonomous Difference Equations, Izvetsiya Akad Nauk Repub. Moldovia Mathematika 26 (1998), 32-42. MR:1659222(99k:58109)

[13] _ A Lyapunov Function for Pullback Attractors of Nonautonomous Differential Equations, Conference 05, Electronic Journal of Differential Equations, 2000, pp. 91-102. MR $1799047(2001 \mathrm{k}: 37020)$

[14] P. E. Kloeden, H. Keller, and B. Schmalfuß, Towards a Theory of Random Numerical Dynamics, Stochastic Dynamics (H. Crauel and M. Gundlach, eds.), Springer, Berlin, Heidelberg, New York, 1999. MR1678487(2000d:37064)

[15] D. E. Norton, The Fundamental Theorem of Dynamical Systems, Commentationes Mathematicae Universitatis Carolinae 36 (1995), 585-597. MR1364499 (97a:58110)

[16] G. Ochs, Weak Random Attractors, Report Nr. 449, Institut für Dynamische Systeme, Universität Bremen, 1999.

[17] C. Robinson, Dynamical Systems. Stability, Symbolic Dynamics and Chaos, 2nd ed., CRC Press, Boca Raton, 1999. MR1792240 (2001k:37003)

[18] K. P. Rybakowski, The Homotopy Index and Partial Differential Equations, Springer, Berlin, Heidelberg, New York, 1987. MR910097 (89d:58025)

[19] D. Salamon and E. Zehnder, Flows on Vector Bundles and Hyperbolic Sets, Transactions of the American Mathematical Society 306 (1988), no. 2, 623-649. MR933310 (89f:58112)

[20] J. F. Selgrade, Isolated Invariant Sets for Flows on Vector Bundles, Transactions of the American Mathematical Society 203 (1975), 359-390. MR.0368080 (51:4322)

[21] G. R. Sell, Nonautonomous Differential Equations and Dynamical Systems. I. The Basic Theory, Transactions of the American Mathematical Society 127 (1967), 241-262. MR0212313 (35:3187a)

[22] Nonautonomous Differential Equations and Dynamical Systems. II. Limiting Equations, Transactions of the American Mathematical Society 127 (1967), 263-283. MR0212314 $(35: 3187 \mathrm{~b})$

[23] , Topological Dynamics and Ordinary Differential Equations, Van Nostrand Reinhold Mathematical Studies, London, 1971. MR0442908 (56:1283)

Department of Mathematics, University of Augsburg, D-86135 Augsburg, Germany

E-mail address: martin.rasmussen@math.uni-augsburg.de 